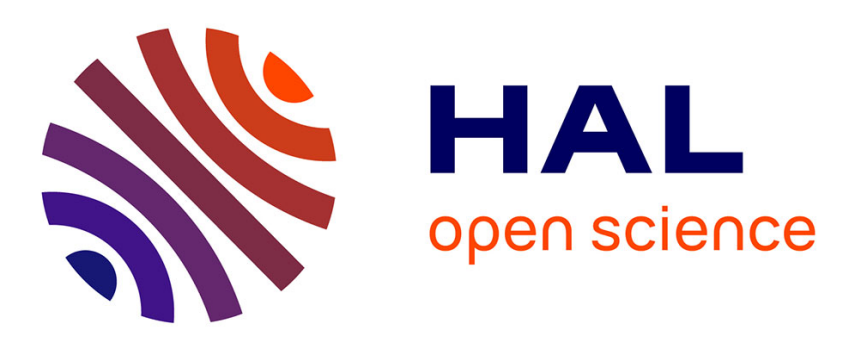

\title{
A gap in Nisbett and Wilson's findings? A first-person access to our cognitive processes
}

Claire Petitmengin, Anne Remillieux, Béatrice Cahour, Shirley

Carter-Thomas

\section{- To cite this version:}

Claire Petitmengin, Anne Remillieux, Béatrice Cahour, Shirley Carter-Thomas. A gap in Nisbett and Wilson's findings? A first-person access to our cognitive processes. Consciousness and Cognition, 2013, 22 (2), pp.654-669. 10.1016/j.concog.2013.02.004 . hal-00940422

\section{HAL Id: hal-00940422 \\ https://hal.science/hal-00940422}

Submitted on 31 Jan 2014

HAL is a multi-disciplinary open access archive for the deposit and dissemination of scientific research documents, whether they are published or not. The documents may come from teaching and research institutions in France or abroad, or from public or private research centers.
L'archive ouverte pluridisciplinaire HAL, est destinée au dépôt et à la diffusion de documents scientifiques de niveau recherche, publiés ou non, émanant des établissements d'enseignement et de recherche français ou étrangers, des laboratoires publics ou privés. 


\title{
A gap in Nisbett and Wilson's findings?
}

A first-person access to our cognitive processes

Claire Petitmengin ${ }^{1,2}$, Anne Remillieux ${ }^{1}$, Béatrice Cahour ${ }^{3}$, Shirley Carter-Thomas ${ }^{1,4}$

1. Institut Mines-Télécom (Télécom EM). 9, rue Charles Fourier, 91011 Evry, France.

2. Archives Husserl, Ecole Normale Supérieure. 45, rue d'Ulm, 75005 Paris

3. CNRS Institut Mines-Télécom (Télécom ParisTech), 46, rue Barrault, 75634 Paris Cedex 13, France.

4. LATTICE (CNRS). 1 rue Maurice Arnoux, 92120 Montrouge, France

\author{
Anne Remillieux \\ anne.remillieux@free.fr
}

Béatrice Cahour

beatrice.cahour@telecom-paristech.fr

Shirley Carter-Thomas

shirley.thomas@telecom-em.eu

Corresponding author:

Claire Petitmengin

claire.petitmengin@telecom-em.eu

tel: (33) 238332760

fax: (33) 160764286

Petitmengin C., Remillieux A., Cahour B., Carter-Thomas S. (2013). A gap in Nisbett and Wilson's findings? A first-person access to our cognitive processes. Consciousness and Cognition 22 (2), 654-669. http://dx.doi.org/10.1016/j.concog.2013.02.004 
Abstract

The well-known experiments of Nisbett and Wilson lead to the conclusion that we have no introspective access to our decision-making processes. Johansson et al. have recently developed an original protocol consisting in manipulating covertly the relationship between the subjects' intended choice and the outcome they were presented with: in 79,6\% of cases, they do not detect the manipulation and provide an explanation of the choice they did not make, confirming the findings of Nisbett and Wilson. We have reproduced this protocol, while introducing for some choices an expert guidance to the description of this choice. The subjects who were assisted detected the manipulation in $80 \%$ of cases. Our experiment confirms Nisbett and Wilson's findings that we are usually unaware of our decision processes, but goes further by showing that we can access them through specific mental acts.

\section{Keywords}

Awareness; Choice blindness; Decision-making process; Elicitation Interview; Explicitation interview; First-person; Introspection; Lived experience; Pre-reflective experience; Passive memory.

\section{Introduction}

One of the most quoted papers in the field of social sciences (almost 7000 citations in January 2013), written in 1977 by Nisbett and Wilson (Nisbett \& Wilson, 1977), draws from a series of experimental studies the conclusion that we have no introspective access to our cognitive processes, notably our decision-making processes: "There may be little or no direct access to higher order cognitive processes" (p. 231). The experiments of Nisbett and Wilson consist in manipulating the cause of the behavior of a subject, and then asking him to explain his behavior: most subjects provide an explanation without noticing the manipulation. The authors conclude that subjects "tell more than they can know" about themselves and describe processes that in fact they cannot access. This work has significantly contributed to the discredit of introspective methods, whose reliability and use in Cognitive Sciences is currently the object of an intense debate (Varela \& Shear, 1999; Jack \& Roepstorff, 2003; Jack \& Roepstorff, 2004; Overgaard, 2006; Petitmengin, 2009a; Petitmengin, 2011; Weisberg, 2011).

A Swedish team of cognitive scientists recently tried to challenge the conclusions of Nisbett and Wilson (Johansson \& al., 2005) by carrying out the following experiment: the 
experimenter shows the participants two pictures of women's faces and asks them to choose which one they find the most attractive. Immediately after, he shows the chosen picture again and asks them to explain the reasons for their choice. But in some cases, the picture which is re-presented is the one that was not chosen (through the use of a double-card ploy, the subject does not realize the manipulation). Surprisingly, the participants detect the substitution in only $27 \%$ of cases, and in $73 \%$ of cases, provide an explanation for the choice they did not make, a phenomenon that the authors term "choice blindness".

Furthermore, a contrastive analysis of various linguistic markers (certainty, specificity, emotionality, complexity...) shows no significant difference between verbal reports corresponding to manipulated and non manipulated trials. This similarity leads the authors to conclude that even in the absence of manipulation, subjects verbalize what they think or believe to have done, not how they actually proceeded, which they are not aware of. The study was repeated a second time by the same team with similar results (Johansson \& al., 2006). It therefore seems to confirm the conclusion of Nisbett and Wilson that we have no introspective access to our decision processes. More specifically, this study casts doubt on the very possibility of remembering our past or just past experience, which is one of the cornerstones of introspection. As Stuart Mill had indeed noted, "a fact may be studied through the medium of memory, not at the very moment of our perceiving it, but the moment after: and this is really the mode in which our best knowledge of our intellectual acts is generally acquired." (Mill, 1882/1961, p. 64). All the introspectionist schools of the early twentieth century - that of Titchener (Titchener, 1910/1980), that of Binet in Paris (Binet, 1903), as well as the school of Würzburg in Germany - agreed on the fact that retrospection must be used each time the introspective act could disturb the target experience. However in the frequent cases where "introspection is retrospection" (Sully, 1881), how can we claim to introspect, for example the reasons for our decisions, if even the memory of the alternatives of the decision is so fragile that we can easily be fooled about them, and thus describe the reasons for a choice we did not make, without even noticing it?

Our practice of interview methods aimed at eliciting the disciplined description of the lived experience associated with a given cognitive process, led us to question the results of these studies. We reproduced the experiment of Johansson and colleagues, whilst introducing an "elicitation" stage for some choices. In the trials where subjects did not undergo an elicitation interview, our results are similar to those of Johansson and colleagues. However, in the trials 
where subjects were assisted in the elicitation of their experience, the substitution was detected in $80 \%$ of cases.

In a first part of this article we will describe our protocol and its differences with that of Johansson et al. After presenting our results in a second part, we will try in a third part to understand them: what did the subjects who were guided in the elicitation of their choice do that allowed them to detect the substitution, and that the others did not?

\section{Protocols and methods}

\subsection{Johansson et al. protocols}

In their first experiment (Johansson \& al., 2005), Johansson and colleagues showed pairs of grayscale pictures of female faces to 120 participants (70 women) and asked them to choose which face in each pair they found most attractive. In some trials, immediately after their choice, the experimenter re-presented the chosen face and asked the participants to state the reasons behind their choice. Unbeknown to the participants, in certain trials, the experimenter used a double-card ploy to covertly exchange one face for the other, and instead presented the one that was not chosen.

Each subject completed a sequence of 15 choices, and was asked to justify his/her choice for 6 pairs, three of which were manipulated. The manipulated face pairs and the non manipulated reports always appeared in the same position in the sequence. The deliberation time and the similarity of the face pairs varied. For time, three choice conditions were included: one with two seconds of deliberation time, one with five seconds, and one where participants could take as much time as they liked. For similarity, two sets of faces were created, a high-similarity (HS) and a low-similarity (LS) set.

Surprisingly, only $13 \%$ of the M trials were detected immediately. The subjects were then given different possibilities to detect the substitution retrospectively. All forms of detection combined, $26 \%$ of manipulated trials were detected. However, once a first detection was made, the participants became more suspicious, and more likely to detect future substitutions. To avoid such cascading detection effects, it was necessary to discard all trials after the first detection was made. This correction reduced the detection rate down to $8,2 \%$ (immediate detection) and 20,4\% (all forms of detection). In 79,6\% of cases, the subjects provided an explanation for the choice they did not make and never detected the manipulation.

Across all conditions, there were no significant differences in detection rate between the HS and the LS sets. There were no significant differences in detection rate between the two 
second and five second deliberation time conditions (20\%, 16,6\% with correction), but it was higher $(36 \%, 28 \%$ with correction) when participants were given free deliberation time.

Johansson and colleagues reproduced this experiment with another group of 80 participants (49 women) (Johansson \& al., 2006). The protocol was very similar to the previous one, except that a fixed deliberation time of four seconds was given to each participant, and the 15 face pairs were constructed in order to keep similarity constant at an intermediate level.

The overall detection rate for the manipulated trials was roughly equivalent to the results of the first experiment, with $27,5 \%$ of the trials detected. It therefore seemed to confirm the conclusion of Nisbett and Wilson that we have no introspective access to our decision processes.

\subsection{Our protocol}

\subsubsection{The choice stage}

We reproduced the experiment of Johansson et al. as closely as possible, but changed one condition, the way in which some of the verbal reports were gathered. As in the original experiment, each subject had to make 15 choices, and was asked to justify his/her choice for 6 pairs, three of which were manipulated. We used exactly the same grayscale pictures as in the second experiment of Johansson et al., which had been designed in order to keep similarity constant at an intermediate level. We gave the choices which were followed by a verbal report the same position as in the initial experiment: position 7, 10 and 14 for the manipulated trials, position 5,9 and 12 for the non-manipulated trials. We used a free deliberation time, a condition that Johansson et al. had identified as the most favourable to detection.

However among the three manipulated reports, two reports (which we will call "NEL" reports) were obtained in the same way as in the initial experiment, and the other through an "elicitation interview" ${ }^{1}$ ("EL" report). At the beginning of the experiment, the subject is told that in some trials, he will be asked to describe his choice. In the case of NEL reports, the experimenter re-presents the chosen (or manipulated) face immediately after the choice, and asks: "I would like you to look at the picture carefully and to tell me why you chose it". In the case of EL reports, immediately after the choice, the picture is given back to the subject, but face down. The experimenter then conducts an "elicitation interview" in order to get the subject to explain his choice process.

\footnotetext{
${ }^{1}$ For the French "entretien d'explicitation", which is sometimes also translated as "explicitation interview".
} 


\subsubsection{The elicitation stage}

\section{General presentation of the elicitation method}

The "elicitation interview method" was initially developed by Vermersch (1994/2011, 1999, 2009, 2012) and then taken up in the context of cognitive science (Petitmengin, 1999, 2001, 2006; Petitmengin et al., 2009; Braboszcz, 2012; Valenzuela Moguillansky, 2012), as well as in the pedagogical (Maurel, 2009), managerial (Remillieux, 2010; Remillieux et al., 2010), technological (Light, 2006; Cahour et al., 2007; Cahour et al., 2010), clinical (Petitmengin et $a l ., 2007)$ and therapeutical (Katz, 2011) fields. This interview method aims at helping a subject to leave the level of representations and beliefs in order to become aware of the way he really carries out a given cognitive process, and describe it with precision.

When asked to describe a given cognitive process, our natural tendency is to slip surreptitiously from the description of our actual experience toward the verbalization of justifications, beliefs, explanations, generalizations, and abstract knowledge about our experience. Contrary to what Dennett suggested by evoking our unchallengeable authority about our experience (Dennett, 2002; Schwitzgebel, 2007), and in spite of the puzzles raised by the concept of self-deception (Mele, 2001), our experience is not infallible, we can misinterpret the way it appears to us (Petitmengin \& Bitbol, 2009). In the case which interests us - the decision-making process - numerous studies, of which the best known are those of Nisbett and Wilson, have indeed shown that when asked to explain the reasons for our choices and decisions, we tend rather to provide justifications or rationalizations, which substitute themselves for the actual decision criteria. This screening is even more efficient if it is "adaptative", that is if it fulfils a function (Wilson, 2002), in other words provides a benefit. One function of justifications for our decisions is to convince our interlocutors of our merits, in order to try to strengthen our self-esteem. Another possible function is to reduce an internal conflict, which may be of a cognitive or an emotional nature. A cognitive conflict occurs when our ideas or beliefs are inconsistent with one another, triggering an uncomfortable feeling of tension called "cognitive dissonance" (Festinger, 1957; Stone \& Cooper, 2001). A response to this discomfort may be to justify one's action by inventing self-justifications. "The Fox and the Grapes", one of the Aesop's traditional fables, is sometimes used to illustrate the concept of cognitive dissonance: the fox that covets inaccessible grapes explains his departure and reduces cognitive dissonance by pretending that the grapes are too sour (Elster, 1983). Self-justification is notably used to justify unethical decisions, in order to make them more 
acceptable and maintain self-esteem (Mills, 1958; Myers \& Bach, 1976). When the conflict is of emotional nature, justifications are rather dubbed "rationalizations". Rationalization occurs "when the individual deals with emotional conflict or internal or external stressors by concealing the true motivations for his or her own thoughts, actions, or feelings through the elaboration of reassuring or self serving but incorrect explanations." (DSM-IV, 1994). This process has been studied in the psychoanalytic tradition, notably by Freud to account for the explanations given for neurotic symptoms (Freud, 1991), and by Fenichel who distinguished various types of rationalization (Fenichel, 1946).

To sum up, we often deceive ourselves about our decision criteria, and this deceit may have a specific function. Even when we try to "introspect" our decision criteria, we do not become aware of deceiving ourselves, because the confabulated criteria surreptitiously substitute for a precise retrieving of the actual decision experience.

However even when we are lucid about our decision criteria, something eludes us: our decision processes. For example, each time we dress in the morning, even if we know the reasons why we choose between several outfits (this one is more comfortable, more becoming, more suited to my activities of the day), we are largely unaware of the rapid internal operations we realize to make our choice (for example, make an inventory of my activities of the day, visualizing myself in this meeting with this outfit, imagining how my feet would feel after an hour's walking firstly in these new shoes, and then in the old ones...). We are seldom aware of how we proceed to choose, and only retain the outcome of this process, that is to say our choice criteria. This absorption into the object, the content, the "what" of our activity, to the detriment of the process, of the "how", concerns not only our decision processes, but most of our cognitive processes. Whether we are touching, seeing, listening, imagining, remembering, understanding or deciding, whether we are performing a concrete or an abstract activity, a large part of our activity, although "lived through" subjectively, is not immediately accessible to reflective consciousness and verbal description. We experience it, but in an unrecognized or "pre-reflective" way (Vermersch 2000, 2009; Depraz et al. 2003). The most surprising thing is that we are not aware of this deficit of awareness, which is the first obstacle in the way of becoming conscious of them: why should I make an effort to acquire an awareness I do not know I lack?

However this difficulty of access does not mean that our cognitive processes are out of reach. It means that accessing them requires a particular expertise, which must be acquired. 
Surprising as it may seem, we are blind to what is the closest to us, our lived experience, and we must learn to see it. This introspective expertise consists in carrying out specific acts (Depraz et al, 2003; Petitmengin, 2006, 2009b, 2011; Petitmengin \& Bitbol, 2009). The "elicitation interview method" aims at triggering these acts, through specific prompts and questions, in order to help a subject to become aware of the unrecognized part of the process being described.

The first key to the elicitation interview consists of helping the subject to choose a particular occurrence of the cognitive process to be described, which is precisely situated in space and time, and bringing the subject back to this singular experience when he moves away from it towards the expression of comments, justifications, explanations and beliefs corresponding not to what he is experiencing but to what he thinks or imagines or believes about his lived experience (and thus interpreting it rather than describing it).

In many cases, there is a temporal gap between the initial experience and its description. The second key to the interview is thus to help the subject to retrieve or to "evoke" the experience, whether it is in the past or only just over (Vermersch 2004/2011, Vermersch 2009, Petitmengin 2006). Evoking a past experience is a very specific process. It falls within a type of memory which Husserl called "passive memory" (Husserl, 1925/2001, Vermersch 2004a, 2004b, 2006). We are always in the process of memorizing what we live, but often involuntarily, without being aware of memorizing. For example you did not voluntarily memorize the first thought you had when you woke up this morning. But you may be able to remember it. As the process of passive memory unfolds unbeknownst to us, we do not know what we know. This explains that at the beginning of an elicitation interview, people usually begin by saying "I do not know what I did, I do not remember anything." However we can turn ourselves toward our past experience to make this information reappear. Interestingly, in passive memory the recalling of the memory also is often involuntary: it occurs spontaneously, usually through the intermediary of a sensorial trigger (Gusdorf 1951). For example, to retrieve the first thought you had when you woke up this morning, you would probably have no way other than returning in thought to your bed at the moment when you awoke. Therefore in the course of an elicitation interview, even if the process to be explored is very fresh, because it has just been carried out, the interviewer helps the subject to retrieve precisely the visual, auditory, tactile and kinaesthetic, and possibly olfactory sensations associated with the very start of the process: "What were you seeing, hearing, feeling... at this 
moment?". The subject "evokes" this moment when he recalls it to the point that the past situation becomes more present for him than the present situation is.

A set of objective - verbal, non-verbal and para-verbal - criteria for checking the effectiveness of the act of evocation have been identified (Vermersch 2004/2011, Petitmengin 2006). The verbal indicators are the use of the word "I", specific context indicators of place and time, the concrete and detailed character (as opposed to conceptual and general) of the vocabulary used. The present tense is often a clue that the subject is re-enacting his experience, but it may also be a clue of generality ("usually, I do this"). An example of a non-verbal indicator is the direction of the eyes: the shifting and unfocusing of the eyes, i.e. the fact that the subject drops eye contact with the interviewer and looks off into empty space, is a clue that the subject is accessing his inner world, ignoring the non verbal reactions of the interviewer which could distract him from this task. At the same time, the flow of speech slows down, the words are often interspersed with silences, and co-verbal gestures often appear (Petitmengin, 2006; Hendricks, 2009): these clues show that the subject is retrieving his past experience and coming into contact with its pre-reflective dimension. It is only when, thanks to these clues, the interviewer verifies that the evocation state is sufficiently intense and stabilised that he can enable the interviewee, with the help of appropriate questioning, to turn his attention towards his inner processes and describe them. It is however rare for the interviewee to remain in the evocation state throughout the interview. Sometimes an ill-advised question or reformulation on the interviewer's part, or an external noise, can be sufficient for the interviewee to lose contact with the past experience. One technique enabling the interviewer to refresh the evocation state consists of formulating questions about the context of the past process, to which the subject cannot reply without "going back to it". Another way is to ask questions about the content or the characteristics of the evocation during the interview, which the subject cannot answer without "refreshing" the evocation.

However evoking the experience is not sufficient to produce a comprehensive and detailed description of it. The third key to the interview consists of helping the subject to redirect his attention from the content, the "what" of his evoked cognitive activity, towards the involved process, the "how" (in our experiment, from the pictures and choice criteria towards the choice process). The diachronic dimension of the process corresponds to the stages of its unfolding in time: the succession of actions, perceptions and inner states which are lived. The synchronic dimension of the process corresponds to its configuration at a given moment in time, which cannot be described under the form of a succession: it includes in particular the 
type of attention and the sensorial modalities which are mobilized (Petitmengin 2001, 2006). To collect these diachronic and synchronic descriptions, the interviewer asks questions which guide the interviewee's attention towards the various moments of the evoked process, without suggesting any content (Vermersch 2004/2011, 2009). This type of "content-empty" questioning enables the researcher to obtain a precise description without imposing his own presuppositions. The structure of an interview is an iterative structure which consists of bringing the subject to evoke again his experience several times, while guiding his attention towards a diachronic or synchronic mesh which is finer each time, until the required level of detail is reached. It is very important to note that it is the reiteration of the act of evocation which ensures the reliability of the description.

\section{Use of the elicitation interview in our protocol}

In our protocol ${ }^{2}$, the interview is conducted as follows: the experimenter / interviewer starts by encouraging the subject to retrieve the moment where the pictures were presented to him: "I suggest you go back a few seconds ago, to the moment when I showed you the pictures. To do this, I propose that you listen again to my voice when I ask the question "which of the two faces do you prefer?", and that you see again the pictures as you saw them then." When the subject shows that he has arrived at this moment (a nod, a smile), the interviewer helps him to retrieve the different phases of his choice process, through questions that "point to" the different moments of the process, without inducing any content, such as: "When you see the pictures, what happens? (...) What happens after this?". The same type of questions is repeated for each phase, in order to elicit a description of a finer and finer diachronic granularity. For example, if the subject says: "First I look at the face on my right", the interviewer asks: "When you look at the face on your right, what do you look at first? How do you go about looking at it? And then?". The interviewer draws the subject's attention more specifically to the moment of the decision and the criteria of choice through questions such as: "At the time when you finally pointed at this face, how did you know that you had made your choice? What had happened just before?".

Whenever the subject contributes new information, the interviewer helps him to deepen his description, through questions that draw his attention to the different - sensorial, attentional and emotional - dimensions of his experience at that particular moment, thus leading him to give a synchronic description, again without inducing the content of the responses. For

\footnotetext{
${ }^{2}$ See Appendix 1 for examples of interviews, with and without explicitation.
} 
example, if the subject says "I started by looking at her eyes", the interviewer draws his attention to his mode of observation: "At that time, when you are looking at her eyes, how do you do this? Are you only focused on the eyes, or do you perhaps see the whole face?".

Throughout the interview, it is the question "how" which guides the subject towards the description of more and more detailed elements of his evoked choice process. While in Johansson et al.'s protocol, the only question is: "Why did you choose this picture?", in an elicitation interview, the question "why", which deflects the subject's attention to the description of explanations and abstract considerations, is never asked (Vermersch 2009). Rather, each time the subject escapes surreptitiously into explanations or generalities about his choice processes, the experimenter brings him back firmly to the evocation of the singular decision process he has just experienced.

Moreover regularly during the interview, and sometimes at the very end of the interview, the subject is invited to refresh the evocation of the moment of his/her choice through injunctions such as "Take the time to retrieve this moment... these photos...", or through questions concerning the content or the characteristics of his evocation during the interview, for example for a visual evocation: "What do you remember best in these photos?", "While imagining these photos, where do you see them (at the top, at the bottom, to the right, to the left)? How far away are they? How big are they now?".

\subsubsection{The post-interview stage}

At the end of the interview (which lasted from 17 to 45 minutes), the interviewer invites the subject to turn over the picture and asks him if "anything else concerning his choice process comes back to him when seeing this picture again."

In the case of explicit signs of detection during the second presentation of one of the manipulated pictures, the experiment is interrupted - in order to avoid cascading detection effects - and we go directly to the debriefing stage of the manipulation (see below). The trial is classified as "immediate detection" (ID).

In case of absence of any immediate signs of detection, as in Johansson and colleagues' protocol, the subjects are given different possibilities to detect the substitutions retrospectively. At the very end of the experiment, the participants are asked the following questions: "What did you think about the experiment?" and "Did you find anything odd about the experiment?". They are then explained the manipulation, and asked: "Did you notice that some of the pictures I gave you back did not correspond with those you had chosen?". Then we ask them to indicate which faces they felt could have been manipulated, first without 
seeing them (which led them to indicate, for example: "This was when you asked me a lot of questions," or "This was the person who had earrings"), then by picking up the manipulated picture(s) in the set.

Four levels of retrospective detection were defined according to the type of reaction of the subject. A retrospective detection of level 1 (RD1) corresponds to the case where just before or after the revelation of the trick, the subject spontaneously and immediately says that he noticed something unusual about the picture in question, and identifies and designates the picture clearly.

A retrospective detection of level 2 (RD2) is the case where after the revelation of the trick, the subject says he noticed something but does not evoke specifically the manipulated picture, and then goes on to indicate it in the set without any false positives ${ }^{3}$, or with false positives, but indicated with a much lower degree of certainty.

In the cases of retrospective detection of levels 3 and 4 (RD3 and RD4), the subject says he noticed something and then finds the picture with false positives, or says he noticed nothing but finally finds it:

- without any false positive or with false positives he indicates with a much lower degree of certainty (RD3).

- with false positives and/or showing other significant clues of detection (during the experiment or during the post-test interview) (RD4).
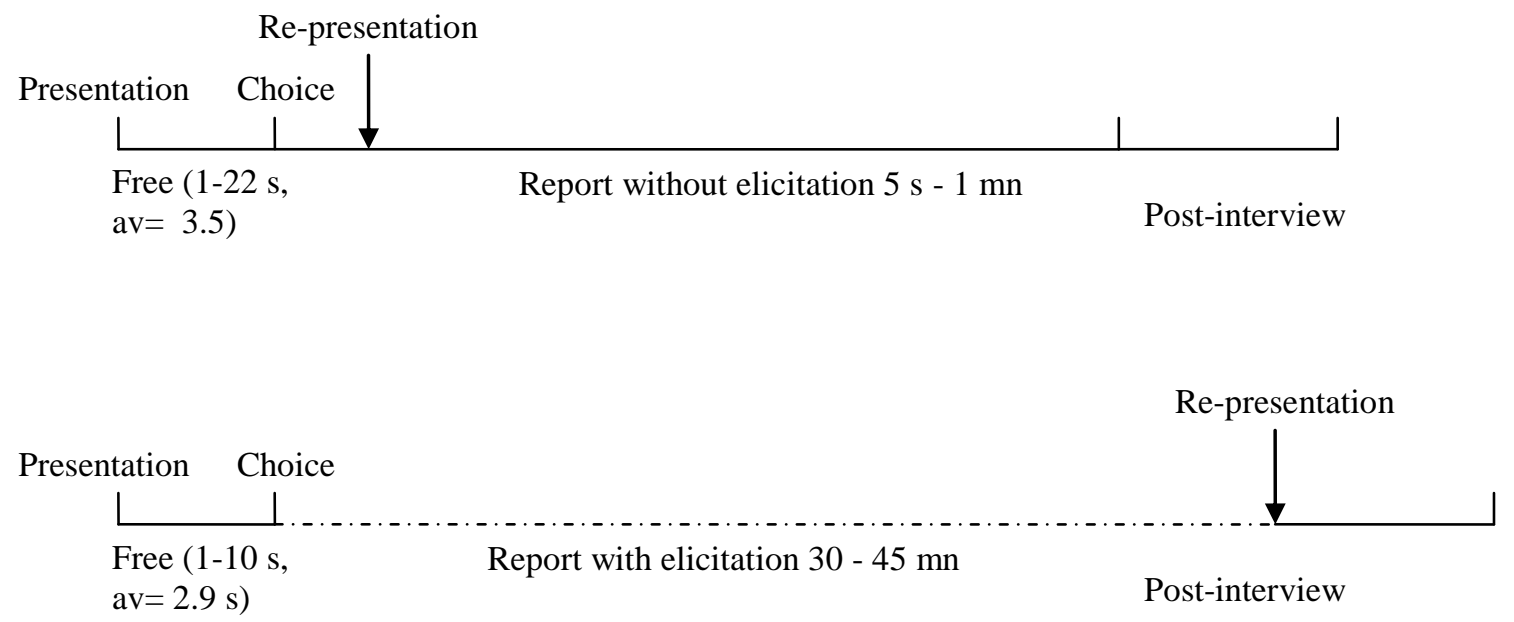

Figure 1 Organisation of the trials with and without elicitation

\footnotetext{
${ }^{3} \mathrm{~A}$ "false positive" is a picture which is indicated although it was not one of those manipulated.
} 


\subsubsection{Additional details on our protocol}

In order to homogenize the chances of detection of the substitution between the elicited and non elicited choices, we implemented the following devices.

- From one interview to the other we varied the pairs of pictures presented, so that for each pair, the proportion of presentations was the same for the choices with and without the elicitation interview.

- We also varied the position of the choice which was followed by an elicitation interview $\left(n^{\circ} 7,10\right.$ or 14 out of 15$)$, in order to prevent, among other possible biases, the two following problems. On the one hand a "learning" bias: to do the interview systematically in first place might make the subject more attentive to his choice processes, which would increase the probability of detection for the following manipulated choices without elicitation. On the other hand a "trust " bias: if the elicitation interview always occurred last, the frequent absence of detection after the previous choices might lull the subject into a false sense of security which would reduce the probability of detection at the end of the elicitation interview.

- In the case of the NEL reports, when the experimenter re-presented the chosen (or manipulated) face immediately after the choice, he asked: "I would like you to look at the picture carefully and to tell me why you chose it". We added this incentive to look carefully at the picture in order to prevent a possible attentional bias: having the elicitation interview before the second presentation might make the subject more attentive to the photo, so that there would be a greater probability of detection in this case.

- We considered the possibility of having the interviewer conduct the interview without knowing which cards were manipulated, but finally decided against this option. The main reason was that we wanted to reproduce the conditions of Johansson's initial experiment as closely as possible, in order to evaluate the one condition which was modified, namely the type of interview. In Johansson's experiment, the cards were manipulated and the interviews conducted by the same person. To realize a blind experiment, the manipulation of the cards and the interview would have to be done by two different persons, which would change the conditions of the trick and thus possibly the detection rate, in the NEL as well in the EL condition.

Moreover, we believe that a blind protocol, which assigns the functions of "interviewer" and "cards manipulator" to two different persons, would not be relevant for our experiment. If there is risk of induction, this risk concerns more the manipulator, who cannot for technical reasons ignore which test he is manipulating, than the interviewer. How in fact could the 
interviewer bias the results of the experiment during the interview? Obviously he cannot influence the choice of the picture, since this choice has been made before the interview. However could he incite the subject to detect the manipulation at the end of the interview? This could conceivably be done in two ways.

The first way would be to give the subject some indications about the content of the picture he actually chose. However we checked carefully that the interviewer, while encouraging the subject to remember his/her choice process, did not make any suggestions regarding the content of the choice (the faces), which would have biased the detection of the manipulation. It should be stressed too that taking care to avoid inducing the interviewee's responses in terms of content - while helping him/her to realize the act of remembering - is a key principle of any elicitation interview. As all the interviews were recorded, the respect of this principle could easily be checked in the interview transcripts, such as the one which is provided in the annex.

The second way for the interviewer to induce the detection of the manipulation would be to communicate nonverbally during the interview his wish for the interviewees to detect the manipulation at the end of the interview. In fact, the interviewer's aim in this case is bound to lead the interviewer to do everything to ensure that the interviewees remember their choice process, which amounts to applying the elicitation interview method as defined in the experimental protocol as conscientiously as possible. In other words, in the specific case of our protocol, as the very goal of the interview is to trigger the acts which enable the detection of truth, it makes no sense to try to prevent this.

30 people (17 women) were interviewed according to this protocol. 141 reports were collected and analyzed, among which 70 "manipulated" reports, including 46 NEL reports and 24 EL reports. The interviewees were management or engineering students who were not trained in elicitation techniques.

\section{Results}

\subsection{Quantitative analysis}

\subsubsection{Analysis of the detection rates}

In this section, we first study detection rates depending on the EL/NEL condition (i.e. with or without an elicitation interview). The effect of the position of the trial on the detection rate is studied in a second stage. For comparison, global statistical indices (Chi-square and Cramer's $\mathrm{V}^{2}$ ) were used to highlight association between variables. We also drew up contingency 
tables to evaluate relative deviations (RD) in each cell of the tables (see Bernard, 2003) in order to examine the weight of the different modalities of the variables. This second procedure is particularly well suited to comparing the different types of detection depending on experimental factors.

In figures 2 and 3, the upper part shows results as detection rates in order to allow a visual comparison as well as a comparison with the results of Johansson et al' (Johansson \& al., 2005). The lower part shows the corresponding raw frequencies.

\section{Global dependency of detection type to elicitation interview}

The global statistical comparisons indicate a powerful impact of the EL/NEL condition on the detection outcome $\left(\mathrm{Chi}^{2}(3)=18.50 ; \mathrm{p}<.001 ; \mathrm{V}^{2}=0.35\right)$. As presented in Figure 2, when taking into account all types of detection, $80 \%$ (20/25) of the EL trials resulted in detection ${ }^{4}$, against only 33\% (16/48) for the NEL trials. In this respect, for NEL trials, the detection rate was close to that obtained by Johansson et al. under comparable conditions: $36 \%$ (28\% corrected) in the first experiment with the "free deliberation time" condition (Johansson \& al., 2005) and $27.5 \%$ in the second experiment (Johansson \& al., 2006).

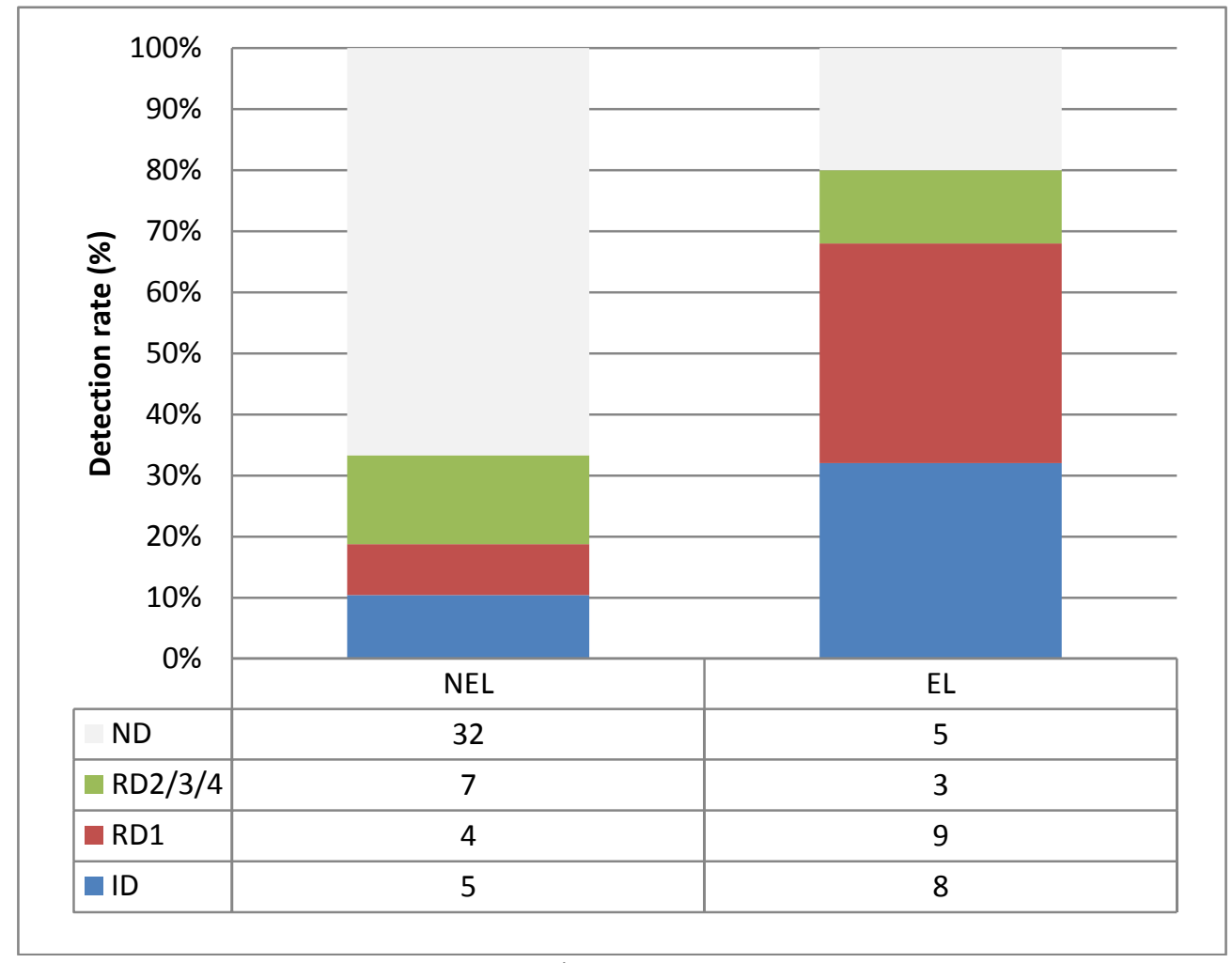

Figure 2 Detection rate and frequencies with / without an elicitation interview

\footnotetext{
${ }^{4}$ The statistical significance of this outcome has been tested by the p-value method, with a null-hypothesis $1 / 3$ for the probability of detection. The calculated p-value for obtaining 20 detections or more in a sample of 24 is 7 . $10^{-7}$. The result can then be considered as highly significant
} 


\section{Details on detection types based on relative deviations}

Contingency tables enable an estimate to be made of the local association between modalities of variables with relative deviations (RDev). RDevs are calculated by comparing each cell of the table - the observed frequencies - with calculated expected frequencies (i.e. those would have been obtained if there was no association between the variables). The association is positive (i.e. there is an attraction) when the RDev is positive; and negative (repulsion) otherwise. By convention, we report only attractions with values $>0.20$. RDevs highlight which modality of a variable (v1), tends to be more associated to which modality of another variable (v2).

\begin{tabular}{|l|c|c|c|c|}
\hline & ID & RD1 & RD2/3/4 & ND \\
\hline NEL & -0.415 & -0.532 & 0.065 & 0.315 \\
\hline EL & 0.797 & 1.022 & -0.124 & -0.605 \\
\hline
\end{tabular}

Table 1 Contingency table of relative deviations for detection type and EL/NEL condition

As shown in Table 1, the ID rate is particularly significant for EL trials $(32 \%$, RDev $=0.797)$ and it is specifically weak for NEL trials $(10 \%$, RDev $=-0.415)$. The same deviation is observed for retrospective detection of level 1 (RD1) with a strong frequency of RD1 for EL trials $(36 \%$, RDev $=1.022)$ and a weak frequency for NEL trials $(9 \%$, RDev $=-0.532)$. Conversely, the ND rate is small for EL trials $(20 \%, \mathrm{RDev}=-0.605)$ and large for NEL trials $(77 \%$, RDev $=0.315)$. But in the RD2/RD3/RD4 condition, the detection rates are not significantly different $(12 \%, \mathrm{RDev}=0.065$ for EL trials and $14 \%, \mathrm{RDev}=-0.124$ for NEL trials). These detailed comparisons indicate that the differences are more substantial in the case of earlier signs of detection rather than late signs.

\section{Effect of the position of trials}

The position of the trial also had an effect on the detection outcome with regard to the EL/NEL factor $\left(\mathrm{Chi}^{2}(15)=27.78 ; \mathrm{p}<.05 ; \mathrm{V}^{2}=0.30\right)$. Figure 3 and table 2 show the differential effect of EL and NEL trials on detection rates depending on their position. For EL trials, position $1\left(\mathrm{P} 1\right.$, trial $\mathrm{n}^{\circ}$ ) specifically enhanced ID $(\mathrm{RDev}=0.872)$ and $\mathrm{RD} 1(\mathrm{RDev}=$ 2.120), with no RD2/3/4 and only one ND. For NEL trials, it is position 3 (P3, trial $\mathrm{n}^{\circ} 14$ ) that is different from the two others, with no ID and no RD1 (for both: RDev = -1). In other words, the elicitation interview enhances earlier signs of detection at the beginning of the experiment whereas the absence of an elicitation interview makes detection very rare at the end of the experiment. We will come back to these results in the discussion section. 


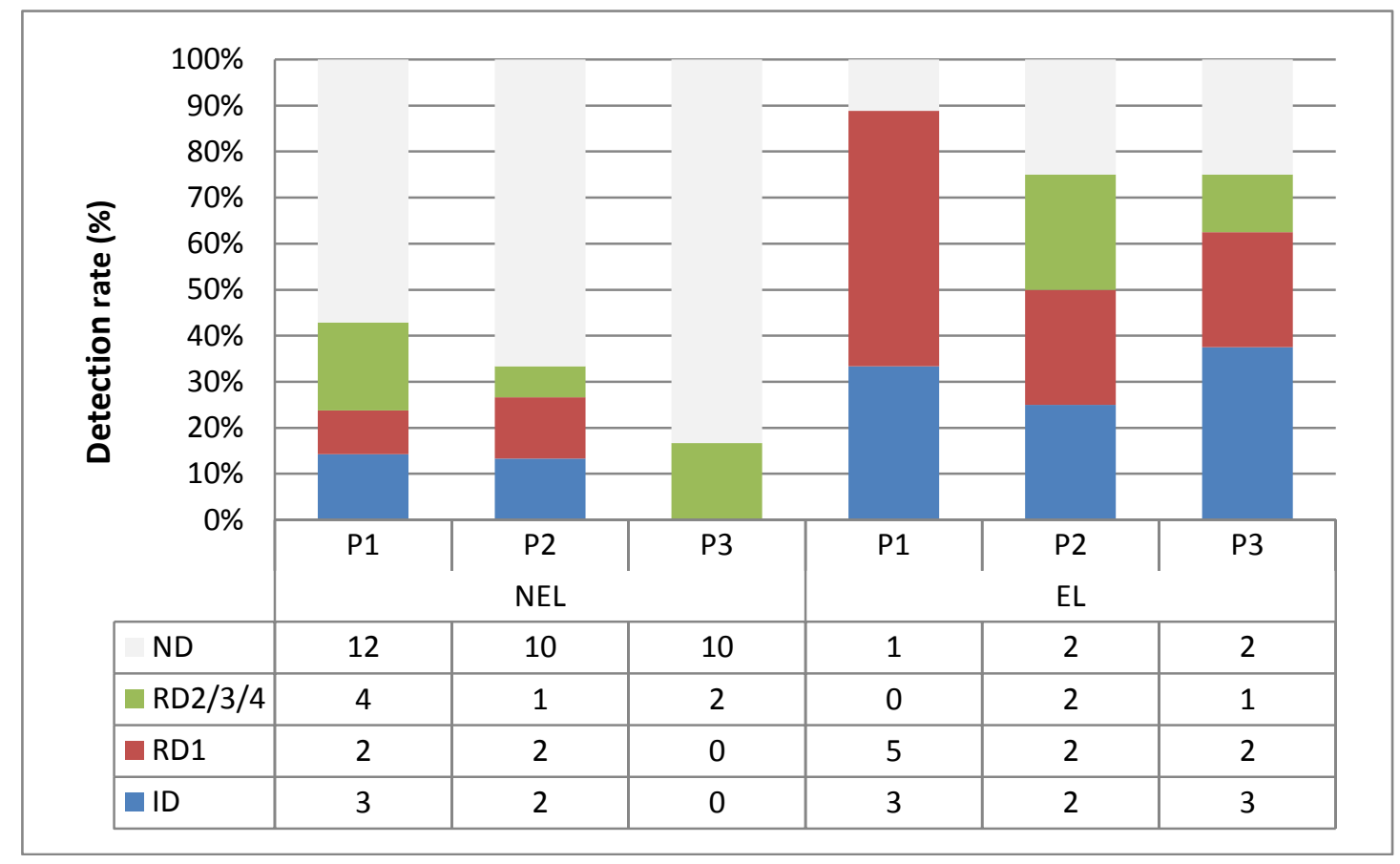

Figure 3 Detection rates according to type and position of the trials

\begin{tabular}{|c|l|c|c|c|c|}
\cline { 3 - 6 } \multicolumn{2}{c|}{} & ID & RD1 & RD2/3/4 & ND \\
\hline \multirow{3}{*}{ NEL } & P1 & $-0,198$ & $-0,465$ & 0,390 & 0,127 \\
\cline { 2 - 6 } & P2 & $-0,251$ & $-0,251$ & $-0,513$ & 0,315 \\
\cline { 2 - 6 } & P3 & $-1,000$ & $-1,000$ & 0,217 & 0,644 \\
\hline \multirow{3}{*}{ EL } & P1 & 0,872 & 2,120 & $-1,000$ & $-0,781$ \\
\cline { 2 - 6 } & P2 & 0,404 & 0,404 & 0,825 & $-0,507$ \\
\cline { 2 - 6 } & P3 & 1,106 & 0,404 & $-0,088$ & $-0,507$ \\
\hline
\end{tabular}

$\mathrm{P}=$ position of the manipulated trial in the sequence of choices

$\mathrm{P} 1=$ position $1\left(\operatorname{trial~}^{\circ} \mathrm{7}\right), \mathrm{P} 2=$ position $2\left(\right.$ trial $\left.^{\circ} 10\right), \mathrm{P} 3=$ "position $3\left(\right.$ trial $\left.\mathrm{n}^{\circ} 14\right)$

Table 2 Contingency table of relative deviations for detection type, EL/NEL and interview position

\subsubsection{Analysis of the deliberation times}

We also conducted an analysis of the deliberation times ${ }^{5}$ for the 72 manipulated choices. The results are the following:

- The average deliberation time is $3.3 \mathrm{~s}$.

- If we take all the trials into account, the detection rate is higher for longer deliberation times: the average deliberation time is $6 \mathrm{~s}$ for the (13) choices which are followed by an immediate detection, and $4 \mathrm{~s}$ if we take all types of detection into account (36 choices),

\footnotetext{
${ }^{5}$ To compare means, we should have the same number of people in each group, and at least twenty people in each group, which is not the case. Nevertheless the comparisons revealed some interesting results.
} 
whereas it is only $3 \mathrm{~s}$ for the (37) choices which are not followed by detection. These results are in line with those of Johansson et al. who found a higher detection rate when participants were given free deliberation time (Johansson \& al., 2005).

- Although the conditions of deliberation are strictly identical, in the EL trials the average decision time $(2.9 \mathrm{~s})$ is lower than in the NEL trials $(3.5 \mathrm{~s})$. Furthermore the average decision time of the choices which are followed by an immediate detection is $3.1 \mathrm{~s}$ in the EL trials, and $10.8 \mathrm{~s}$ in the NEL trials. These differences in average deliberation times between EL and NEL trials are explained by the presence of two NEL trials with particularly long decision times (16 s and $22 \mathrm{~s}$ ), which can only be explained by chance. If we do not include these two trials, the average decision time in the NEL trials is $2.8 \mathrm{~s}$, that is very similar to the average decision time in the EL trials $(2.9 \mathrm{~s})$. And the average decision time in the NEL trials that led to immediate detections is $5.3 \mathrm{~s}$, which means that the only NEL trials which led to immediate detections ( 5 out of 48 ) started with long deliberation times.

- Deliberation times for NEL trials preceding and following the EL trial are identical (3.5 s). We will come back to these results in the discussion section.

\subsection{Comparison of the verbal reports with and without elicitation}

We conducted a detailed comparative analysis of both types of reports, EL and NEL.

The first finding is that the EL reports are much longer than the NEL reports: 2900 words versus 208 words on average. The analysis then shows that the NEL reports are focused on the criteria of choice: facial features and/or feeling aroused ("the one on the right is smiling," "she has more delicate features," "I find her friendly"). On the other hand, the EL reports contain, in addition to the criteria of choice, descriptions of the observation and choice processes. Subjects describe how they went about watching the faces, comparing them and deciding. For example: "I look at her globally, then I zoom on the mouth", "I imagine how she would react if I said hello", "I compare by keeping the first face in mind and superimposing it on the second like a tracing".

A preliminary linguistic analysis of EL and NEL reports reveals some significant differences which tend to confirm these observations. Pronoun distribution, for example, is very different. The proportion of verbs which are introduced by the pronoun "I" is proportionally twice as high in EL reports (60 versus 28 per 1000 words) reflecting the fact that the descriptions are primarily focused on the subjects' own choice processes. Conversely the number of occurrences of the pronouns "she" and "they" is almost twice as high in the NEL 
reports (21 versus 12.5 per 1000 words), in which the descriptions are essentially focussed on the characteristics of the faces. The reflexive character of EL reports is further confirmed by the much greater number of reflexive verbs, indicating a switch in the subjects' attention towards their internal processes (6.1 versus 1.7 per 1000 words): "je me dis" (I say to myself), "je me demande" (I ask myself), "je m'assure" (I make sure) ${ }^{6}$, "je me focalise" (I focus my attention), "je m'imprègne" (I let myself become permeated by"). The actionfocused character of the descriptions is also confirmed by the greater variety of verbs in the EL as opposed to the NEL reports (104 versus 33 different verbs), with nearly all the 71 extra verbs in the EL reports being action verbs (for example: I start, I stay, I go back, I stop, I look for, I compare, I keep, I stay). Interestingly, the French verb "faire" (to do/make/perform an action) does not appear even once in the NEL while it appears 73 times in the EL, for example: "je fais des comparaisons" (I make comparisons), "je me fais une image dans ma tête" (I make a picture in my head), "je fais des aller-retours (I go back and forth).

We also performed an analysis of the content of the EL reports. Such an analysis aims to identify the structure of the experiences described, that is a network of relationships between descriptive categories, independently of the experiential content, and to detect any generic structures which are gradually extracted from the initial descriptions thanks to a succession of abstraction operations (Petitmengin, 1999; Petitmengin \& Bitbol, 2009). This enabled us to identify three types of choice strategy: immediate or non-immediate, positive or negative, "features-focused" or "feeling-focused" strategy.

1) The "immediate" choice (9 trials / $20^{7}$ i.e. $45 \%$ ) is made easily, whereas in the "nonimmediate" choice (11 trials / 20 i.e. 55\%), the subject has trouble deciding. This subjective evaluation of the difficulty of choice and decision time is consistent with the objective decision time (an average of 1.2 seconds for the immediate choices and 4.5 seconds for the non-immediate choices).

2) The subject uses a positive strategy ( 9 trials / 18 i.e. $50 \%$ ) when he reaches a decision based on the face he prefers, and a negative strategy ( 9 trials / 18 i.e. $50 \%$ ) when he decides

\footnotetext{
${ }^{6}$ In some cases the English equivalent does not require the reflexive pronoun to indicate a reflexive meaning.

${ }^{7}$ The trials which were difficult to classify according to a particular strategy were not taken into account. This explains why the total number of trials is sometimes inferior to 24 .
} 
on the basis of the eliminated face. The (positive or negative) choice criteria are either the features of the face or the feeling aroused by it.

\begin{tabular}{|l|l|l|}
\hline & "Features" criteria & "Feeling" criteria \\
\hline $\begin{array}{l}\text { "Positive" } \\
\text { strategy }\end{array}$ & $\begin{array}{l}\text { "The face is smoother, finer features, } \\
\text { more pleasant and regular. " } \\
\text { "The hair and hair color ... the gaze ... } \\
\text { the nose and mouth, and the face shape } \\
\text { as well. " } \\
\text { "She really had a little smile ... that's } \\
\text { what has reinforced my choice. " } \\
\text { "There is this lock of hair on the side } \\
\ldots \text { which I liked. It was more natural, } \\
\text { less overdone... the lock of blond } \\
\text { hair." }\end{array}$ & $\begin{array}{l}\text { "Maybe I felt closer to her. I am slim too and so ... } \\
\text { since she is also slim... I say to myself we have } \\
\text { fomething in common. I don't say it too much in } \\
\text { more of a general state of my body ... I feel ... } \\
\text { slim. I saw it was with her that ... I have more in } \\
\text { common so I chose her. " } \\
\text { "I wondered who was the one I felt the closest to, } \\
\text { who I would have more confidence in. Perhaps } \\
\text { also the person who I would most likely go and } \\
\text { talk to, and exchange with afterwards, who would } \\
\text { reassure me the most. " }\end{array}$ \\
\hline $\begin{array}{l}\text { "Negative" } \\
\text { strategy }\end{array}$ & $\begin{array}{l}\text { "The choice was made on the eyes. It } \\
\text { is after having noticed ... well, the } \\
\text { irregularity of the eyes of the person } \\
\ldots \text { that I did not choose her. I thought } \\
\text { that I would choose this one. " }\end{array}$ & $\begin{array}{l}\text { This [selected] faced shocked me, appealed to me } \\
\text { less than the other. I chose it because the other } \\
\text { made me think, well ... made me think of someone } \\
\text { else, in fact, I chose this one by eliminating the } \\
\text { other. " }\end{array}$ \\
& \multicolumn{2}{|l}{} \\
\hline
\end{tabular}

Table 3 Positive and negative strategies criteria

3) The "features-focused" strategy (5 trials / 22 i.e. $22.7 \%$ ) consists in exploring and comparing the features of the faces, the way we play the "spot the seven differences game" (a metaphor that appeared in four interviews).

"One remembers the look, the shape of the nose, of the face, that we have seen, and one tries to see the similarities and differences between the face we have just seen and the face we see now, to compare, after say 'well, as a result, there, which of the two, which one I preferred.' "

Some participants describe a chronology of exploration of the features ${ }^{8}$ :

"The eyes, the position of the nose, the mouth, and then the limit of the chin."

"If I had to represent the course of my gaze, it was the hair, then it went down uh ... the opposite side from the kind of strand of hair she had, then went up, yes, that's it. [The way I went round the face] is precisely in a clockwise direction ..."

"There is the quick look, then there is the hair, then I look at the hair of the picture next, and then I go down, yeah, and so on. "

The mode of attention is visual and focused: the features are "scrutinized" one by one.

"Once one has seen that the nose was not straight bah one will zoom. I look at her nose ... well I finally confirm that she has something wrong. "

"I see the mouth and also that there are small wrinkles, expression wrinkles. The rest I don't see it very well. I'm only interested in the smile. "

"It's as if I was zooming on the mouth."

\footnotetext{
${ }^{8}$ A line of objective validation of this type of description would be to compare them with recordings of eye movements: when the subject says he starts by looking at the picture on the left, does he really proceed this way?
} 
The comparison is carried out either by rapid back and forth movements between the two pictures, or by "superimposing" the two faces.

\begin{tabular}{|c|c|}
\hline $\begin{array}{l}\text { Alternative } \\
\text { observation } \\
\text { of the faces }\end{array}$ & $\begin{array}{l}\text { "I go back and forth between the two pictures." } \\
\text { "There it's really looking at the details, then coming to the other one and ... it's going } \\
\text { back and forth, in fact, between the two photos. " } \\
\text { "I juggled between the two pictures ... I looked at the hair, comparing with the other, } \\
\text { it's a checklist: that, it's done. That's done... and I go to the next point and so on. " } \\
\text { "In order to scan all the criteria that I may have, the differences, as in the differences } \\
\text { game a bit, when you're a child and you play the differences game, so I try to play the } \\
\text { differences game. I think I look at them in turn, quickly. I don't think I look at them at } \\
\text { the same time ... to find differences I don't look at the same time, I look on the left, } \\
\text { then on the right, for example, is this one tired, has she dark rings under her eyes? } \\
\text { And the other girl, has she dark rings under her eyes? Is she wearing makeup? And } \\
\text { the other girl, has she got makeup on? " } \\
\text { "First I will look at one picture and then another, well, I'll return from one photo to } \\
\text { the other ... very quickly we'll say." }\end{array}$ \\
\hline $\begin{array}{l}\text { Mental } \\
\text { superimposition } \\
\text { of the faces }\end{array}$ & $\begin{array}{l}\text { "They were linked in my memory like that, to make the decision, like a tracing. Inside } \\
\text { my head." } \\
\text { "And I compare while keeping the first one in memory. It is superimposed as a layer. } \\
\text { I have the picture in my head and I visualize the picture that I'm looking at and the } \\
\text { one I remember, it appears like with the tracings kids in kindergarten do and so I } \\
\text { superimpose a layer and I look at the points, the seven differences game. " } \\
\text { "It was... between brackets, two images that I superimposed. It's like taking a tracing } \\
\text { paper and saying ... well, I have drawn the main features and ... if I superimpose the } \\
\text { two tracings, well, it works. " } \\
\text { "It's easier to compare when they are one upon the other and this really allows me to } \\
\text { have both together and as a result it's easier to compare. [I really put them] one upon } \\
\text { the other, that is to say that I will, for example, put the eyes on the eyes, and now ... } \\
\text { really on the hand [location where the picture has been observed]. " } \\
\text { "It allowed me to see that it was thinner, because in the juxtaposition the other face } \\
\text { was wider, its outline went over that of the first face." }\end{array}$ \\
\hline
\end{tabular}

Table 4 "Features-focused" strategy

In the latter case ("superimposing" the two faces), the comparison requires a "visual retention", that is to say the pre-reflective and involuntary creation of a more or less complete mental image of at least one of the two faces, which is superimposed on the picture being observed.

"The visual image remains in the short term, remains imprinted... temporarily."

"I had the image of the previous one in my head."

"I keep it in memory. It remains in my head. I have her in my mind but it is a little fuzzy compared to the picture I am watching. It is fuzzy compared to the picture I have in front of my eyes. "

On the other hand, the "feeling-focused" strategy (6 trials / 22 i.e. $27.3 \%$ ) consists in evaluating successively not the features of the faces but the feeling or emotion aroused by each, and then comparing them. 
"I had a general impression. The first thing, was the emotion, well the feeling."

"I look and it will inspire something to me. I need to have a sensation, to feel something before [comparing]. [I look at the picture] more for the sensation. It's more the look that emanates from her when one looks at her, is not even really looking at her, it's something which happens when you look at her, how you feel the person. "

To do this, the photograph is "personified":

- the face is put into context, that is to say imagined as part of a body and sometimes in a specific location;

- an interaction, or rather the hint of a possible interaction, for example an exchange of glances, is imagined;

- the reaction (the desire to talk to the person, or on the contrary a feeling of unease or fear) is evaluated.

\begin{tabular}{|c|c|}
\hline $\begin{array}{l}\text { Perception of the } \\
\text { face as a person } \\
\text { (as opposed to its } \\
\text { perception as a } \\
\text { photo) }\end{array}$ & $\begin{array}{l}\text { "I had the impression she was wearing a hooded sweatshirt, like me, her hair was } \\
\text { pulled back as if she was playing sports, not necessarily made up or anything. She had } \\
\text { a hood. I thought, but I thought, well, very, very subtly that she was a sporty person, } \\
\text { one can feel that she's a sporty person. " } \\
\text { "I try to imagine approximately her corpulence. I imagined, with the shape of her } \\
\text { face, it was a somewhat corpulent person. But ... with a round face, quite pleasant. I } \\
\text { try to imagine approximately her style of dress. Then, I imagined her clothes ... not } \\
\text { ample, but quite feminine, but ... not tight, pretty ... I thought they went well with her } \\
\text { face and hair." } \\
\text { "The moment I look at her eyes, I rather see a person, since I think she is sad... it } \\
\text { reminds me of feelings and therefore, she is a person. I'll try to understand why she } \\
\text { feels that way. I try to put myself in her situation. Why, me, would I feel like that. I } \\
\text { try to go back to memories of ... events that may have affected me or made me ... like } \\
\text { her. " }\end{array}$ \\
\hline $\begin{array}{l}\text { Contextualisation } \\
\text { of the person }\end{array}$ & $\begin{array}{l}\text { "I see them at work, yes ... well I see them in the hall of offices, a bit like here in fact. " } \\
\text { "I imagine her queuing at the bakery, looking less made-up a little as if she had just } \\
\text { jumped out of bed on Saturday morning. " } \\
\text { "I just imagined them in the subway" } \\
\text { "I would imagine them well in the street, with people wandering in a street, rather a } \\
\text { busy street with ... activity. Not a wide boulevard, a large avenue, but a medium-sized } \\
\text { street in Paris. " }\end{array}$ \\
\hline $\begin{array}{l}\text { Imagination of an } \\
\text { interaction }\end{array}$ & $\begin{array}{l}\text { "I imagine would I go towards her easily. I imagine myself talking to her, easily. I } \\
\text { imagine actually myself being in front of her and her answering me. It happens, there } \\
\text { is an exchange. She seems to look at me and she's not cold. I feel there may be an } \\
\text { interaction. } \\
\text { "(I see) something angular and a little hard, something closed, which doesn't let me } \\
\text { come in. Something that is not favorable to a relationship or exchange or circulation. } \\
\text { " } \\
\text { "(I feel) I step back, I shrink back. It's not disgust, (but) a step backwards, (in) the } \\
\text { legs, a step back, the right foot which wants to step back, not the left but the right. " } \\
\text { "I imagine how she would react, in fact, if I did that, if for example I said hello, what } \\
\text { would she, how would she react, how would she smile." }\end{array}$ \\
\hline
\end{tabular}


This strategy is associated with a mode of attention which is global, defocused: the face or the person is observed as a whole; and receptive: it is a matter of letting oneself be permeated by what "radiates" from the person.

"I looked globally. One sees the whole face ... I can still describe it, it was quite round, she looked blonde, light eyes, I see the entire photo. I saw actually the picture as a whole."

"I look at the overall appearance ... in a holistic manner. "

"I'm not going to focus on anything in particular but really on the whole and what emanates from it. I see her, the global expression. "

"I start with the overall look, well what emanates from the photo, well from the person. "

"I see the face in the background, I am focused on the smile but I see the face in the background. It's not as if the smile took all my vision, I see in the periphery the rest of the face and of the picture. "

"Retention" of one of the faces may be used during the observation of the other, but in this case it is not a visual retention but a retention of the feeling which is aroused (a "felt retention").

"It comes back with the overall impression I might have of the person before. I already have an a priori about the person I looked at. Basically it's as if I could still hear a little voice in my head telling me, 'yes, but with the other you got a different impression', it comes to confirm what I might have felt before. It's not a voice, it's more a kind of feeling, an intuition. Not even in my head actually. That would be there... in my heart. "

In 11 trials out of 22 i.e. $50 \%$, the subject uses a mixed strategy, combining a featuresfocused and a feeling-focused strategy. All these strategies sometimes include an internal criterion informing the subject of the fact that his decision has been made (sense of relief, of determination...).

"I feel better. I feel that this may be a relief. (...) It is something comfortable in fact, the feeling of: 'it suits me'. It calms me down in fact. (...) Even the way of breathing ... I have this feeling... that I breathe ... well that I breathe out more (easily) in fact."

"Determination, (...) I am convinced, it is: "That's it, I know it, that's all. It is this one". (...) And I do not regret it later."

The analysis of the interviews also revealed a variation in the detection rate depending on the type of strategy used, an additional result to which we will return to in the discussion.

\section{Discussion}

In summary, without elicitation, the subjects only detect the substitution in $33 \%$ of the cases, while the picture remains hidden to them for 2 seconds. At the end of an elicitation interview, the detection rate is $80 \%$ while the picture remains hidden during the whole of the interview, that is 17 to 45 minutes. How can this discrepancy be explained? What do the subjects do in this case, which explains why they detect the substitution much more frequently, while the 
time between the initial observation of the photo and the presentation of the trick photo is much longer, which should greatly reduce the chances of detection?

This experiment was especially designed so as not to trigger a specific effort of memorization at the moment of the choice, through particular instructions aimed at making the events more salient at that time (Nisbett \& Wilson, 1977, p. 251). As Johansson et al. explain, "this experiment was meant to simulate a choice situation in which no prior evidence indicates that a high level of monitoring is needed" (Johansson et al., 2005, Supporting Online Material p. 4 and 10). It corresponds to situations of everyday life where we do not voluntarily memorize the alternatives nor the reasons for our choices, the situations that are the most common.

The conditions of memorization in the EL and NEL trials are identical in this respect. The difference in detection rates cannot therefore be explained by a greater stimulus salience at the moment of the choice in the EL condition, which would explain better memorization, and consequently slower memory decay than in the NEL condition. The subject never knows if the choice he is making will be followed by an elicitation interview or not. Moreover as we have shown in section 3.1.2, the average deliberation time is lower in EL trials $(2.9 \mathrm{~s})$ than in NEL trials ( $3.5 \mathrm{~s}$ ), and in trials which result in immediate detections (ID) it is more than three times shorter in the EL trials (3.1 s) than in the NEL trials (10.8 s). Thus the higher detection rate in the EL trials cannot be explained by a special effort of memorization at the moment of the choice in anticipation of the interview, which would have resulted in a longer decision time.

In other words the higher detection rate cannot be explained by slower memory decay. On the contrary, the fact that the delay between the two presentations of the pictures is much longer in the EL condition would predict a much lower detection rate - the longer the delay the lower the detection rate. The fact that this rate is much higher, which cannot be accounted for by a difference in the memorization of the faces at the moment of the choice, would only seem to be explained by a specific and efficient act of remembering at the time of the report.

If we go on to consider the detection rates according to the position of the trials (figure 3), we observe that they are higher in the first manipulated trial than in the second and third trials, in the EL condition as well as in the NEL one. This decrease in detection rates between the first and the second trial shows that the experiment did not trigger a "learning" effect: the interviews did not generate a greater effort of memorization in the subsequent trials, which would have resulted in higher and higher detection rates in the course of the experiment. The 
absence of learning effect is confirmed by the fact that the average deliberation time remains stable throughout the experiment. However this decrease in detection rates may be explained by an interference effect (Peterson \& Peterson, 1959): the increasing number of faces which have been seen in the course of the experiment may make it more and more difficult to remember the two faces which have just been seen. The earlier detection in the EL trials as well as the higher detection rates in both types of trials in the first position, could be explained by the absence of this effect at the beginning of the experiment. But while the detection rate decreases dramatically in the third NEL trial, it remains stable in the second and third EL trials. This continuous decrease in the NEL trials could be explained by a "trust" effect, in addition to the interference effect: the absence of detection in the two first trials may have generated a sense of security, which resulted in a sharp fall of the detection rate. However the trust effect, as well as the interference effect, do not occur in the EL condition, indicating that they are counterbalanced by a specific activity during the interview.

These observations lead us to conclude that the difference in detection rates between the EL and NEL trials cannot be explained by a difference in the memorization of the faces at the moment of choice, but by differences between the EL and NEL conditions at the time of the report.

The main differences between the results in the EL and NEL conditions are the following:

(1) higher detection rate in the EL condition;

(2) far more detailed descriptions of the choice processes in the EL condition.

The main differences between the conditions of the reports are the following:

(a) in the EL condition, subjects are periodically asked to recall the pictures and the moment of the choice;

(b) in the EL condition, more questions are asked.

From these conditions and results, we draw the following conclusions:

(A): (1) is due to (a). It is the fact that participants are periodically asked to recall the pictures and the moment of the choice which causes the higher detection rate in the EL condition.

(B): (2) is due to (b). It is the large number of questions which triggers detailed descriptions of the choice processes in the EL condition.

From (A) "The fact that participants are periodically asked to recall the pictures and the moment of the choice causes the higher detection rate in the EL condition", we deduce (A'): "The fact that participants are periodically asked to recall the pictures and the moment of the 
choice in the EL condition induces an act of recall of this moment, which triggers the detection of the manipulation."

The recall instructions still enabled the participants to detect the manipulation after forty minutes of interview in $80 \%$ of cases (this much longer gap between the two presentations of the pictures might have led to a much poorer detection rate). This shows that the act of recall was still efficient after that time gap. We can therefore conclude (C) that the descriptions of the choice processes given in the course of the interview relied on reliable memories of the moment of the choice, which strongly bears out the validity of these descriptions.

From (B) "It is the large number of questions which triggers detailed descriptions of the choice processes in the EL condition", we deduce (B'): "The large number of questions induces an orientation of the subjects' attention towards their choice processes which causes detailed descriptions of these processes in the EL condition."

We consider that these acts of recall and orientation of attention are the introspective acts which enable the participants to provide a description of their choice processes which is both detailed and reliable.

Our hypothesis is that in the NEL reports, the subjects do not perform any of the above acts. On the one hand, they do not evoke the moment of the choice. One of the reasons which may explain this absence of evocation is the social interaction in which they are engaged, which does not incite them to introspect. They simply provide a justification for their choice that makes use of current publicly available information and makes sense in the context of the current social interaction, which is based on an implicit relationship of trust in the interviewer. We hypothesize that the subjects content themselves with justifying their choice by commenting in real time the photograph they have in front of their eyes - which explains why Johansson et al. found no significant difference between the manipulated and nonmanipulated reports. The implicit trust in the interviewer, as well as the question "why", do not prompt them to retrieve precisely the moment of the choice, which might have led them to detect the manipulation. The memory has not faded, it is simply not summoned up. The viewing of the video recordings of the two types of reports supports this hypothesis: during the NEL reports, subjects look alternately at the interviewer and at the picture they have in front of them. On the contrary, during the EL reports, the subjects are encouraged to evoke the moment of the choice, which leads them to break off eye contact with the interviewer and 
look "into empty space" at the evoked pictures (in a location which may be different from the table, where the pictures were originally shown), this unfocusing of the eyes being one of the criteria of the evocation state. The loss of eye contact contributes to the creation of another type of social interaction, where the interviewer is very present through his prompts and questions, while at the same time authorizing the subject to "leave" the usual mode of interaction in order to come into contact with his internal processes - which enables him to detect the manipulation. In other words, in the NEL condition the (inauthentic) relationship of trust and the persistence of eye contact with the experimenter make it difficult for the participant to imagine that he could have been deceived, while the EL condition fosters the participant's confidence in his own processes, which enables him to recognize the manipulation.

On the other hand, subjects in the NEL condition do not redirect their attention towards their choice processes. They keep focused on the characteristics of the faces they have in front of them, i.e. on criteria (of the choice they did not make). In the absence of any introspective skill or help of the interviewer, they are not aware that they have done anything particular in order to choose, nor that something particular may be done to retrieve it. Our hypothesis is that this absence of redirection, coupled to the absence of evocation, can explain the paucity of the reports, in addition to their unreliability.

Furthermore the absence of a "learning" effect - the fact that the detection rates in the NEL trials following an EL report do not increase - seems to confirm that it is not sufficient to carry out these acts of evocation and redirection once in order to know how to go about doing them, and that they are genuine skills which need to be learned.

It is true that a few NEL trials result in detections. However, the fact that these detections are preceded by especially long deliberation times (an average of 11 seconds while the general average time is 3.4 seconds) suggests that the very scarce detections in the NEL condition are due to a stronger memory trace due to more time spent examining the face at the moment of the choice, and not to an act of evocation of the faces during the report.

But how can we explain the fact that after the elicitation interview, some subjects detect the substitution and some others do not? Should the descriptions which did not lead to detection be considered as inauthentic? Among the four descriptions which did not lead to detection, three are particularly detailed, rich and above all consistent. This consistency, defined as the absence of contradiction and the complementarity and coherence of various elements of 
description within the report, is in our opinion a strong criterion of authenticity. As the elicitation interview is an iterative process, the report it results in is a jigsaw made up of scattered pieces of descriptions, which the analysis aims to piece together again. When this reconstitution results in a coherent sequence, it is hard to imagine that the initial description could have been constructed by the subject without the guiding thread of lived experience. This raised the question as to whether the lack of detection could be explained by the nature of the strategy of choice used. To try to answer this, we looked for possible correlations between the detection rate and the type of strategy. This revealed that all the cases of non detection followed a "feeling-focused" strategy, whereas most immediate (7/8 i.e. 87.5\%) and retrospective (9/11 i.e. $81.8 \%)$ detections followed a "features-focused" or mixed strategy. Moreover, all the subjects who instantly detected the substitution (8/8), and $75 \%$ of the subjects who detected it retrospectively (9/12), used retention at the moment of the choice ${ }^{9}$. In 7 trials out of 8 immediate detections, and in all the cases of retrospective detection, this was a visual retention. However among the subjects who did not detect the substitution, only one used retention, and this was a felt retention (see figure 4).

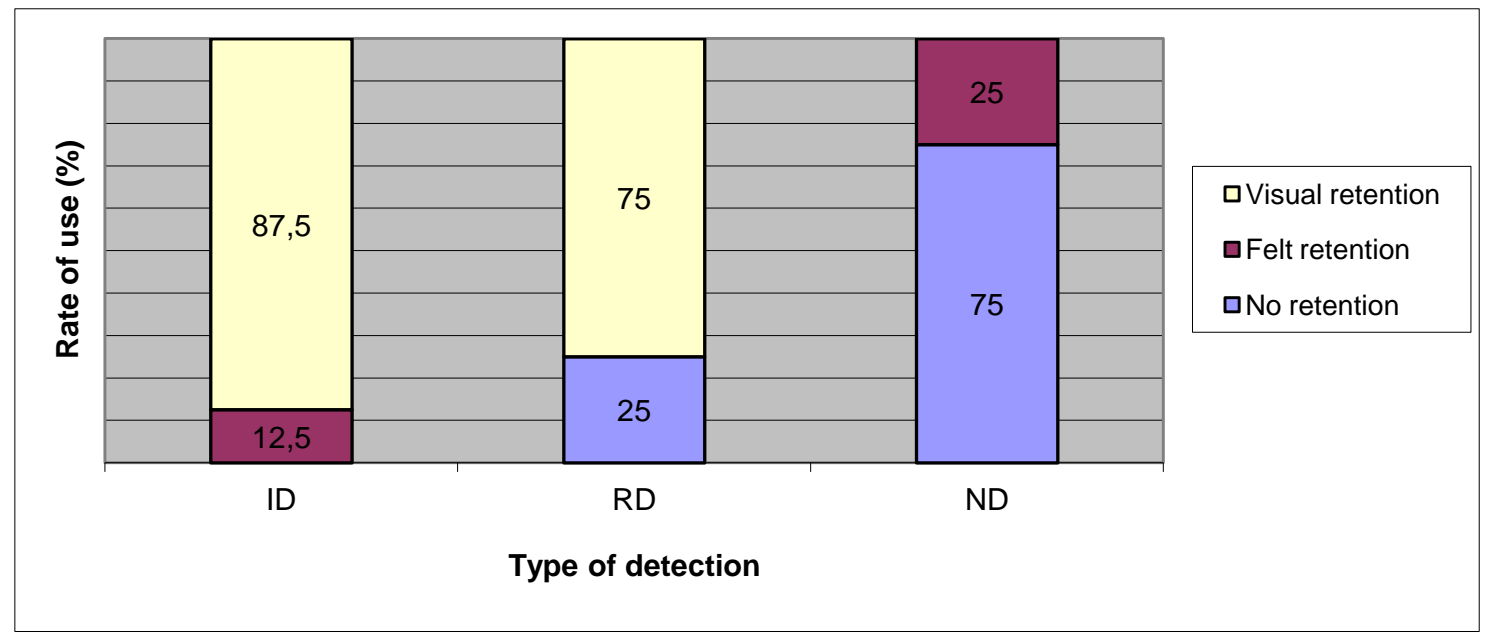

Figure 4 Rate of use of retention per type of detection in the EL condition (ID = immediate detection, RD = retrospective detection, ND = no detection)

The use of visual retention at the moment of the choice, which characterises a featuresfocused or mixed strategy, therefore seems crucial in detecting the substitution. Its absence in the "feeling-focused" strategies might therefore explain why 3 trials out of 4 result in a nondetection. But the low detection rate after the NEL choices, which may be assumed to use the

\footnotetext{
${ }^{9}$ The trials which were difficult to classify according to a particular strategy were not taken into account. This explains why the total number of trials is sometimes inferior to 24 .
} 
same proportion of visual retention as the EL choices, shows that retention is not sufficient in itself. Our hypothesis is that the process of evocation reactivates during the interview the retentions which were used at the time of the choice, and that this reactivation is necessary to retrieve the initial experience and thus to detect the substitution.

\section{Conclusion}

Our experiment confirms that naïve descriptions of our decision-making processes are usually poor and unreliable. However it shows that it is possible to access these processes by carrying out specific acts consisting in evoking the process and directing one's attention towards its different dimensions. These acts make it possible to produce a description of the choice criteria and processes which is both rich and reliable, as evidenced by the high detection rate of the substitution in our experiments. The fact that we knew our reports were reliable meant that we were able to analyze their content and to detect regularities in the form of a set of choice strategies.

The experiments of Nisbett and Wilson, like those of Johansson et al., whose subjects were not using a truly introspective approach, cannot therefore be considered to have discredited in any way the possibility of experientially accessing our decision-making processes in a disciplined way and studying them rigorously and systematically. In this perspective, choice blindness can be considered as a particular case of our ordinary blindness to lived experience, which can be remedied using appropriate means or procedures (Depraz, Varela \& Vermersch, 2003; Petitmengin, 2006, 2009b; Petitmengin \& Bitbol, 2009). This possibility of disciplined description of our decision processes gives access to vast and almost unexplored deposits of data, and opens up several avenues of investigation. The first consists in deepening the "first person" study of decision making: repeating the same protocol with a larger number of subjects, designing protocols involving other types of choices. The second avenue consists in studying in more detail the acts enabling such a disciplined access: their nature, the means that make it possible to elicit them through an interview or possibly selfintrospection, their subjective and objective (notably linguistic) criteria of realization, the evaluation of the level of expertise of the subjects and of the interviewers, the adaptation of introspective techniques to the type of process under study and to the strategy being used. Such studies will lead us to better understand and control the processes which enable us to access our cognitive processes.

\section{Acknowledgments}


We would like to thank Céline Astruc, Michel Bitbol, Tom Froese and Anil Seth for their useful advice and comments. We are also grateful to Dominique Fréard for his help in performing the statistical analyses.

This study was supported by funds from Renault, France.

\section{References}

Bernard, JM. (2003). Analysis of Local or Asymmetric Dependencies in Contingency Tables using the Imprecise Dirichlet Model. Third International Symposium on Imprecise Probabilities and their Applications (ISIPTA'03). Lugano, Switzerland, July 14-17.

Binet, A. (1903/2004). L'étude expérimentale de l'intelligence. Paris: L'Harmattan

Braboszcz, C., (2012). Study of the electroencephalographic correlates of mindwandering and meditation. Université Paul Sabatier, Toulouse.

Cahour B., Brassac C., Vermersch P., Pachoud B., Salembier P. (2007). Etude de l'expérience du sujet pour l'évaluation de nouvelles technologies; l'exemple d'une communication médiée. Revue d'anthropologie des connaissances 1(1), 85-120.

Cahour, B., Forzy, JF., Martin, C. (2010). Feelings and strategies of senior drivers: ways of coping with fear? Proceedings of ECCE 2010 (European Conference on Cognitive Ergonomics). Delft, The Netherlands, August 24-27.

Dennett, D. C. (2002). How could I be wrong? How wrong could I be? Journal of Consciousness Studies 9 (5-6), 13-16.

Depraz, N., Varela, F., Vermersch, P. (2003). On becoming aware. The pragmatics of experiencing. Amsterdam: John Benjamins.

DSM IV. (1994). Diagnostic and Statistical Manual of Mental Disorders. American Psychiatric Press Inc.

Elster, J. (1983). Sour Grapes: Studies in the Subversion of Rationality. Cambridge University Press.

Fenichel, O. (1946). The Psychoanalytic Theory of Neurosis. London: Routledge.

Freud, S. (1991). Case Histories II. London: Penguin Books. 
Froese, T. Gould, C. , Seth, A.K., Validating and Calibrating First- and Second-person Methods in the Science of Consciousness, Journal of Consciousness Studies 18 (2), 40-64.

Gusdorf, G. (1951). Mémoire et personne. Paris: PUF.

Hendricks, M. (2009). Experiencing level: An instance of developing a variable from a first person process so it can be reliably measured and taught. Journal of Consciousness Studies, $16,129-155$.

Husserl, E. (1925/ 2001), Analyses Concerning Passive and Active Synthesis: Lectures on transcendantal logic (Boston, MA: Kluwer Academic Publisher).

Jack, A. \& Roepstorff, A. (Eds). (2003). Why Trust the Subject? Part 1. Journal of Consciousness Studies 10 (9-10).

Jack, A. \& Roepstorff, A. (Eds). (2004). Why Trust the Subject? Part 2. Journal of Consciousness Studies 11 (7-8).

Johansson, P., Hall, L., Sikström, S. \& Olsson, A. (2005). Failure to detect mismatches between intention and outcome in a simple decision task. Science 310 (5745), 116-119.

Johansson, P., Hall, L., Sikström, S., Tärning, B. \& Lind, A. (2006). How something can be said about telling more than we can know: On choice blindness and introspection. Consciousness and Cognition 15, 673-692.

Katz, E. (2011). Attending to Clinical Practice: A Phenomenological Study Exploring the Structure of Clinical Attention and its Relationship with Holistic Competence. PhD thesis, University of Toronto.

Light, A. (2006). Adding method to meaning. A technique for exploring people's experience with technology. Behaviour and Information Technology, 25(2), 175-187.

Maurel, M. (2009). The explicitation interview: Examples and applications, Journal of Consciousness Studies 16 (10-12), 58-89.

Mills, J. (1958). Changes in moral attitudes following temptation. Journal of Personality 26, 517-531.

Myers, D. G. \& Bach P.J.. (1976). Group Discussion Effects on Conflict Behavior and SelfJustification. Psychological Reports 38, 135-140.

Mele, A., 2001, Self-Deception Unmasked, Princeton: Princeton University Press.

Nisbett R.E. \& Wilson T.D. (1977). Telling more than we know: Verbal reports on mental processes. Psychological Review 84, 231-259.

Overgaard, M. (Ed). (2006). Special Issue on Introspection. Consciousness and Cognition 15 (4). 
Peterson, L.R., \& Peterson, M.J. (1959). Short-term retention of individual verbal items. Journal of Experimental Psychology 58, 193-198.

Petitmengin, C. (1999). The Intuitive Experience. Journal of Consciousness Studies 6 (2-3), 43-77.

Petitmengin, C. (2001). L'expérience intuitive. L'Harmattan: Paris.

Petitmengin, C. (2006). Describing one's Subjective Experience in the Second Person. An Interview Method for the Science of Consciousness. Phenomenology and the Cognitive Sciences 5, 229-269.

Petitmengin, C. (Ed). (2009a). Ten Years of Viewing from Within. Journal of Consciousness Studies, 16 (10-12).

Petitmengin, C. (2009b). Editorial Introduction. Journal of Consciousness Studies 16 (1012), 7-19.

Petitmengin, C. (Ed). (2011). Ten Years' Viewing from Within: Further Debate. Journal of Consciousness Studies 18 (2).

Petitmengin, C. (2011). Describing the experience of describing? The blind spot of introspection. Journal of Consciousness Studies 18 (1), 44-62.

Petitmengin, C., Navarro, V. \& Le Van Quyen, M. (2007). Anticipating seizure: Prereflective experience at the center of neuro-phenomenology. Consciousness and Cognition $16,746-764$.

Petitmengin, C. \& Bitbol, M. (2009). The validity of first-person descriptions as authenticity and coherence. Journal of Consciousness Studies 16 (10-12), 363-404.

Petitmengin, C., Bitbol, M., Nissou, J.M., Pachoud, B., Curalucci, C., Cermolacce, M., Vion-Dury, J. (2009). Listening from Within. Journal of Consciousness Studies 16 (10-12), 252-284.

Remillieux, A., Petitmengin, C., Ermine, J.L., Blatter, C. (2010). Knowledge Sharing in Change Management: A Case Study in the French Railways Company, Journal of Knowledge Management Practice 11 (3).

Remillieux, A. (2010). Explicitation des connaissances de conduite du changement à la SNCF : Vers une gestion des connaissances pré-réfléchies, Editions Universitaires Européennes.

Sahdra, B. \& Thagard, P. (2003). Self-Deception and Emotional Coherence. Minds and Machines 13, 213-231. 
Schwitzgebel, E. (2007). No unchallengeable epistemic authority, of any sort, regarding our own conscious experience - Contra Dennett?. Phenomenology and the Cognitive Science 6, 107-13.

Stone, J. \& Cooper, J. (2001). A self-standards model of cognitive dissonance. Journal of Experimental Social Psychology 37, 228-243.

Sully, J. (1881). Illusions of introspection, Mind 6 (21), 1-18.

Titchener, E.B. (1910/1980). A Textbook of Psychology (New-York: Scholars'Facsimiles and Reprints).

Valenzuela Moguillansky, 2012. The relation between pain and body awareness: $a$, investigation using experimental and experiential methods. $\mathrm{PhD}$ thesis, University Pierre et Marie Curie, Paris.

Varela, F.J. \& Shear, J. (Eds). (1999). The View from Within. First-person approaches to the study of consciousness. London: Imprint Academic.

Vermersch, P. (1999). Introspection as practice. Journal of Consciousness Studies 6 (2-3), $17-42$.

Vermersch P. (2000). Conscience directe et conscience réfléchie. Intellectica 31, 269-311.

Vermersch, P. (2004a). Modèle de la mémoire chez Husserl. 1/ Pourquoi Husserl s'intéresset-il tant au ressouvenir. Expliciter 53, 1-14.

Vermersch, P. (2004b). Modèle de la mémoire chez Husserl. 2/ La rétention'. Expliciter 54, $22-28$.

Vermersch, P. (2006). Rétention, passivité, visée à vide, intention éveillante. Phénoménologie et pratique de l'explicitation. Expliciter 65, 14-28.

Vermersch, P. (2009). Describing the practice of introspection. Journal of Consciousness Studies 16 (10-12), 20-57.

Vermersch, P. (2004/2011). L'entretien d'explicitation. ESF: Paris.

Weisberg, J. (Ed). (2011). Describing Inner Experience. Journal of Consciousness Studies 18 (1).

Wilson T. (2002). Strangers to Ourselves: Discovering the Adaptative Unconscious. Cambridge, MA: Harvard University Press. 
«... »: a silence.

"...3"»: a silence of 3 seconds.

\section{GAELLE}

\section{NEL report}

Choice $n^{\circ} 10$

Decision time: 3"

With manipulation

Length of the interview: $36^{\prime \prime}$

1. I'll ask you to return the photo, to look at it carefully, and to tell me why you chose this one.

2. Well there it was a little different, it's true that I found both pretty, but this one, I was attracted by her eyes.

3. By her eyes?

4. Yes... I don't know why because the other one was very, very, very pretty too, but I was attracted by her eyes. This uh... this young girl, from far off in fact it's as if she had eyes... I don't know, clearer that the other one, or more uh... no, maybe they are not clearer, but more, I don't know, they seem more... nicer.

5. Nicer?

6. A nicer gaze, a nicer gaze, and someone who would make me, well, calm, peaceful, someone pleasant, well, I... I like this face ([laughter].

7. Okay. Is there anything else?

8. Uh... No.

\section{RODOLPHE}

\section{NEL report}

Choice $n^{\circ} 5$

Decision time: 8"

Without manipulation

Lenght of the interview: 1' 13"

1. I'll ask you to return the photo, to look at it carefully, and to tell me why you chose this one.

2. Well, it's a comparison [clears his throat]. I have to remember the other one. Well, I... because uh... well, it's the... say the tidiness because the other' person's hair was completely uh... tousled, the hairstyle was very uh... uh...

characteristic... of uh... of a person I would not got on with uh... and the earrings too, I remember they were rather uh... big and uh... not very nice. While uh... well, clearly, in fact, it's clearly the earrings that made me choose uh... this one, yes.

3. The earrings.

4. So in fact, it's the earrings and the hairstyle that made me... and the shape of the jaw too.

5. Okay. Is there anything else, apart from this?

6. The shape of the nose and the fact that... maybe the previous one had a little more weight than this one uh... and this is a rather important characteristic today. Uh... that is, the hairstyle, the hearings, the shape of the jaw. 
1. Now, I'll ask you not to return the photo immediately and go back a few seconds in time to the moment of the very last choice, when I showed you the last two pictures. I would like you to go back to this moment as if you were experiencing it now. Take the time you need. To do this, I suggest that you retrieve the position you had at that time, when I showed you these last two pictures...

2. Hmm

3. Take your time. I suggest that you listen again to my voice when I asked you: "which of these two faces do you prefer?"

4. $\mathrm{Hm} \mathrm{Hm}$

5. And that you let the pictures come back, and see them again as if you were seeing them at that time....

6. $\mathrm{Hm} \mathrm{Hm}$

7. Tell me when they come back. Take as much time as you need. Are they coming back to you?

8. «...»Y Yes.

9. Where do you see them, now?

10. Yes... where, wait...?

11. These photos, these faces, when you think of them again, where do you see them?

12. There and there [gestures].

13. In my hands or not?

14. Yes, in your hands.

15. Do you see them both at the same time?

16. Yes.

17. How big are they?

18. " ... 8" " Quite large. I focused on them uh ... as there is a good definition uh ... on the print, well, I saw them well, the faces are rather large.

19. Compared to the real size they have, do you see them larger or smaller?

20. They are larger.

21. What do you remember best in those faces?

22. Two brunettes at least. «...» And there, the choice was far less uh ... difficult than for the other pictures. One of them was much more delicate uh ... with a much deeper gaze, say uh ... a whiter skin, there was a ... stronger... sharper contrast between the skin and the hair, it was pretty uh ... nice. Uh ... large eyes for this one, the one I chose in fact ... while the other was uh ... rather ordinary.

23. More delicate, with a deeper gaze. How does the other one come back to you? How do you know she is ordinary?

24. Because I chose this one [gesture]. And the other one [gesture], she makes me think a lot of... the pictures I saw before ... which I hesitated about.

25. Do you see it fuzzy or clear? How do you see this photo?

26. Very fuzzy, a brunette... ordinary. Uh ... rather uh ... ordinary, a pretty round face shape, uh ... eyes uh ... well, nothing very special, precisely.

27. So if you agree, we'll try to track back to see how you went about choosing. I would like you to go back to the moment when you are discovering the pictures, at the very beginning, and to tell me what happened at that time. What did you do?

28. I looked at the two faces, first at the nice girl, then at the ordinary one.

29. First you looked at the nice girl. What happened when you looked at the nice girl?

30. I said to myself "the other one would have to have (laugh) something to uh... compete with her" and... I looked at the other and I said to myself that it was not the case then, I chose.

31. How do you go about saying that? How did it happen? Do you hear something? Do you talk to yourself? Do you have any other feelings? How do you go about it?

32. [clears his throat] How do I choose?

33. No, at the very beginning when you were looking at the nice one, you said to yourself: "the other one would have to have something to compete with her ". I wonder what happened to you concretely. How did you think of this? Did you say it in your head or did something else happen?

34. Uh... « ... 4" » No, it's... it's... mostly a reflex.

35. A reflex, okay. This reflex, could you describe it to me. Take your time to go back to this moment...

36. «...»Uh... «... 12" »It's a reflex that uh ... happens more ... well, not everyday, uh ... when you see two people and that... you are rather attracted by one or the other, uh ... just physically, it's something that ... we are used to living every day so uh ... this is not something I said to myself... I did not say "does she uh ..." well, I did not put words on my emotions, it was in fact pure emotions. 
37. It was a pure emotion that you are used to feeling when you meet people and you did not say anything special to yourself . At that time, at the very beginning, when you are looking at her, do you look at a particular part of her face or do you look at her globally?

38. The first thing I noticed was her black hair, her white skin, the contrast, then the eyes, the shape of the jaw ... which is very important, actually ... especially for men, but for women uh ... also and... that's it. So it was rather uh ... from top to bottom, in fact.

39. You first looked at, you said, the hair and the contrast with the...

40. The forehead then ... because the forehead is where there is less shade so that's where you really see the colour of the skin. Then, uh ... the eyes and then, the shape of the jaw.

41. The hair and the forehead, do you look at them at the same time or successively?

42. «... 5"» at the same time.

43. When you look at them, do you also see something else around them or do you only see the forehead and the hair?

44. «... 12" " Uh... I see something around, that is to say that I see the ... uh ... background. I see the light ... that illuminates the face. So uh ...

45. Where do you see the light illuminating her face, is it the whole face or it is a particular part of her face, at that moment?

46. «...» The light comes from the ... left [gesture]. Uh ... I wondered if it was the sun or if it was a light well ... a halogen, an electric light. «...» Uh... « ...»

47. So you look at the hair, the forehead, there is a light coming from the left, you wonder if it is electric or natural. At this point, have you already made your choice, well, do you already find her pretty or not?

48. Yes, I already find her pretty.

49. What do you feel exactly, then, as you look at the top of her face, the contrast between her hair and the skin of her face?

50. [Laughter] Really?

51. Hm.

52. I said to myself it would be ... [clears his throat] nice to have a few more girls like this in the school.

53. You said to yourself it would be nice to have a few more girls like this on the school. When you say that, what is happening inside you? Do you feel something special, a feeling, a voice?

54. Frustration, I think. Precisely, of ... uh ... well, not to meet girls like that, as nice as that.

55. Okay. So then, at that moment, you are looking at her, you say to yourself that it would be nice if there were more of them in the school and you feel a frustration about not meeting ...

56. More girls like that.

57. Okay. What happened after that, just after?

58. «...» Well, as it was about comparing, I compared and the choice was uh ... well, almost ... instantaneous.

59. Okay. Then you compared. How did you go about comparing, at that moment?

60. Well, I did exactly the same manoeuvre as with the other girl. I looked at her face, so, her black hair... a hairstyle a little less uh... natural, I found. What ... that is to say that ... with all these faces that you showed me, that one, I ... I remember the emotions I had, but I don't remember exactly the hairstyle. But I remember that the hairstyle of the second one was less attractive. Uh « ...5" » and ... the forehead, the eyes, already at that time, I was telling myself it was not great, and the shape of the jaw, it was... rather puffed up, rounded [gesture]. And then I ...

61. Okay. So you look at the first one, and then as it is about comparing you do the same manoeuvre with the other one, first you look up, and down, you find the hairstyle less attractive, less natural. And at that moment, is the other face still present or not, while you are looking at the one you have not chosen?

62. Not the face, the emotion.

63. Could you tell me in what form it occurs, where it occurs?

64. «... 5"» I think the word "frustration" is the more uh...

65. Appropriate?

66. Appropriate, yes.

67. Okay. So when you look at the other, you still feel that frustration. If I had this feeling, what would I feel? This feeling, this sensation, is it is a feeling that you can locate in your body or is it a general impression? How do you feel this frustration?

68. «...» Uh... it's something to do with the lungs [gesture towards his chest]. A difficulty in breathing. Uh ... a need to fill one's lungs and sigh.

69. Okay. So it's as if you had trouble breathing, a need to breathe, to fill your lungs.

70. A heaviness, yeah, which is at this level [hand on his chest].

71. Do you experience other things at that moment that makes you feel frustrated?

72. So, is it ... well, because ... my answers are ... are all in a particular context of my life at that moment, so is it this kind of things that you are asking me about , or is it really the millisecond where I was looking uh ... ?

73. Actually, yes, it's the millisecond where you were looking, but if during this millisecond, other pictures came or things that were related to your life, it can be integrated. But it's this millisecond. I do not want explanations, you know what I mean, but if you experienced frustration at that moment, I'm interested in this. Maybe there are other things coming to you about what you felt at that moment?

74. Uh... no, not about the second face, because the choice was already made.

75. Okay. Anyway, when you are watching the one you have not chosen, you still feel this heaviness on the lungs, if $I$ understood correctly 
76. (15 :26) Hmm. Actually, yes, perhaps I felt it. Well, I exacerbate all these things for you to understand, but ... well, so that you can interpret them, but uh ... this feeling lasted a quarter second and ... and it completely evaporated with uh ... when I looked at the second picture.

77. It evaporated at that moment?

78. Yes, I didn't ... I got nothing, I felt nothing at all when I looked at the second picture.

79. Okay. And the first picture, is it still present in some form, at that moment, when you are looking at the other one. So, you just looked at the one you find attractive and then you look at the one which inspired you nothing particular, that you find ordinary. And a moment ago, I asked you if ... the first one was still present and that's when you spoke of frustration. And finally, you are not sure you felt that at that moment?

80. Frustration is when I looked at the first photo. After that, I looked at the second and ... nothing left.

81. Okay. This feeling of frustration, of heaviness in the lungs, at which precise moment does it disappear? Take your time to go back.

82. «...» At what moment, uh ... at the moment when in fact, I go down well ... from the top of the face to the eyes, it's at that moment, I guess. I could not tell you precisely, but I think it is ... at that moment.

83. So, it was still there at the beginning and then it disappears when you scan the face from top to bottom.

84. Yes.

85. And at what point do you make your decision?

86. When I reach the bottom of the second face.

87. Okay, when you reach the bottom of the second face. What happened when you made your decision?

88. «...» I remembered the emotion with the first one and I said to myself that ... well ... obviously what you are asking me, this is, I choose the one that ... arouses something, an emotion and a pleasant emotion. Even if in fact it's unpleasant, she's still nevertheless the prettiest.

89. She's still the prettiest. So, you arrive at the bottom of the face and there, you think again of the emotion which the other one aroused. Are there any other things that come back from the other one? Do you see her again at that moment or not at all?

90. Yes, I remember her forehead and the whiteness of her skin.

91. So at that moment, where do you see her again, at the time when you decide?

92. Where do I see her again? In your hands?

93. The memory, in fact, do you see her again or do you think of her again in your head or elsewhere?

94. "... 7" » It's not the image as such that I saw again, I would say, it's the emotion.

95. It's the emotion that came back. So, you re-experience that emotion, if I understand you well. At that moment, are you still looking at the one you have eliminated, or don't you look at her anymore?

96. No, I don't look at her anymore, because ... I ... scan the first face, which gives me ... an emotion. Then I scan the second one. And then to be sure I come back to the first face and I ...

97. Okay, so you go back to the first face. And when you go back to the first face, what do you do, what do you look at?

98. Eyes, I think, general appearance.

99. Okay. How do you go about looking at general appearance?

100. By indeed looking at the eyes.

101. And how do you go about looking at both eyes and general appearance?

102. In fact, it's ... I think it's the eyes that give life to everything else. As ... I think I remember she had larger eyes than the second. «... ». So ... it seemed to me obvious that she was more ... prettier, because it gave ... her eyes gave more life to her ... her face ... which in itself, had more life than the other girl, since the contrast was stronger.

103. Okay. Then you come back to this one, you look at her eyes, and her eyes gave life to her face. How do you feel they give life to her face?

104. Each pair of eyes has a uh ... a way to ... «...» How can I say this ... there is a certain depth say, and certain way to look. And ... most of the pictures you showed me, uh ... even the girls ... I had chosen, the girls ... uh ... said nothing at all in their eyes and you did not got the impression that they felt an emotion in front of ... the camera.

105. So if I understand well, you feel that this one feels an emotion, her eyes have a depth, they give life to her face. At that moment, do you see her as a photo or as a person?

106. As a person.

107. How do you know that you see her as a person, at this particular moment?

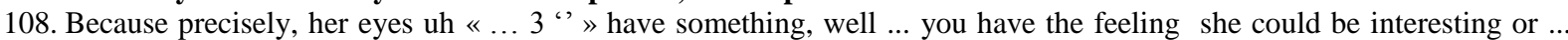
maybe it's just because she's pretty in ... in general terms, that is to say that ... I'm a little confused, because I saw ... only a quarter second, but ... « ...» trying to be clearer, her eyes ... gave depth. One gets the impression they feel something or they could possibly feel something. And you ... well ... now, when I reflect, I'm wondering where she is or... if she is on uh ... a seat or if she is standing.

109. Okay. You are looking for the context around, where she is actually, behind this photo. At the exact moment when you are looking at her a while ago, while choosing, do you imagine her somewhere or not?

110. Yes.

111. Can you describe to me what came to you?

112. I wondered where the camera was located, if it were on a foot or in the hand ... of the photographer. If uh ... it was in a room in Paris or if it was in a ... isolated village hall.

113. Okay. How did you go about thinking about all this? Did you have particular images that came to mind?

114. Yes. Uh ... like ... like a video camera, in fact, which ... which makes a close-up [gesture], on what the camera sees ... and then that ... that would open and would let everything else appear uh ... first the body of that girl and then ... the context uh... of the sheet that hangs behind [gesture]. 
115. Okay. So if I understand correctly, it's as if you had opened the focus of the photo and you see her body, with a white sheet behind, perhaps. Do you see her sitting, standing?

116. Sitting, yes. On a high bar stool.

117. On a high bar stool. Okay. Is there any movement, in this room?

118. Yes. Listen, it's not the opening of a photo, it's the opening of a ... of a film rather.

119. Can you describe the movement to me, what happens? Does she move or is it that other things move?

120. She does not move, no. It's the ... photographer who asks her to remain still.

121. Okay. Can you see the photographer?

122. Yes. A rather tall man, holding the camera, which does not have ... a tripod, in fact.

123. So you see a rather tall photographer, who is taking the photograph. She's rather still, if I understand well. Are you in the scene or not?

124. «...» No. No, it's as if the images were broadcast on a screen, and I was much further.

125. The person, well the girl who is being photographed, which direction is she looking in? Can you see her gaze or not?

126. Yes, because I'm standing behind the photographer and ... and uh ... I see the photographer from behind and I step back while seeing the ... this man from ... from behind ... and thus seeing this girl uh ... a little bit ... further.

127. If I understand well, you step back?

128. Yes.

129. Okay. When do you stop stepping back?

130. "... 5" " I don't stop, because ... there is a window which is [gesture]... on the side, in fact, the ... the focus opens more and more and lets the window appear, because it's the sun that lights up her face. Uh... and then, uh ... then, uh... I stop when I'm back to the wall, say. Because I don't manage to work out ... to imagine where this scene is, if it's in an apartment or ... uh ... in Paris or if it's in a small village.

131. So there is a point where you stop, it's as if you were back to the wall, where you don't manage to imagine the scene, and you stop stepping back. What happens next?

132. «... 11" » In my head or in the scene?

133. In your head.

134. Well, after that I say to myself that it is ... probably ... uh ... a small town, actually. It's certainly not uh ... a big city. Because otherwise, the choice of the other people who are ... well, uh ... all the others who were photographed by the same ... the choice would have been, certainly, uh ... more uh ... although, maybe not ... but all these ... all these ladies ...I was shown, this is really the uh ... appropriate word, I think, ladies. "Ladies", not ... girls or ... young women.

135. Okay. So if I understand correctly, at that time you are still thinking about your photo and the context of... well, how all these girls were photographed.

136. Yes.

137. Okay. So if you agree, I'm going to ask you, now: this scene where suddenly the focus opens, when you imagine the scene of the shot, at which moment is it? Is it at the moment when you saw her for the first time, the one you chose, or is it at the second time that you saw this scene? At which precise moment was it? Take your time, huh, to come back to the moment of the choice, a while ago, when I presented the two photos to you.

138. «... 12" » It was the second time. The first time I had just scanned the face in the same way than ... the other, than all the others. But ... when I came back to it, it let me ... have a little more time to let uh ... my imagination.

139. Okay. So the first time you scan her from top to bottom, and there, you do not see her as a person yet.

140. Well, that's it.

141. And the feeling of frustration, it was the first time you saw her, right?

142. The first time, yes.

143. Okay. Did you also imagine her in a scene or not, I mean the one you eliminated?

144. After having my back to the wall, actually. After the focus has opened, I thought again of all the other ... ladies who ... who were in exactly the same situation as that uh ... pretty girl.

145. Okay. How did you go about thinking of the others, at that moment?

146. Well, I remember ... in particular the two ... well, the first two faces you showed me. Two ladies, in their thirties, a blonde, a brunette. Nothing exceptional about either. One had a flattened jaw and the other one a ... a hollow, there, cheeks which ... were ageing.

147. Okay. And you imagined them in this scene, did you?

148. Yes. I put them in place of this girl and ... and it is at this moment that I thought it was surely uh ... in another city, not ... not somewhere in Paris. Paris is really the place where ... where pretty girls uh ... are numerous.

149. Okay. So you imagine the other girls, the other girls whom you did not especially like, in that place, you think that this ... photo was taken in a rather small town, well, not in Paris.

150. It's what I imagine within a few seconds.

151. So I propose that you see the pictures once more, the last two pictures that I presented to you. Take your time to look at them again, to go back in the situation a while ago, when you discover them. Tell me when they come back. Retrieve the first position you had, you can hear my voice again, when I asked you which of these faces do you prefer.

152. «... 10"» Yes.

153. Where do you see them, in my hands or elsewhere?

154. Still in your hands, the one I chose in this hand and the other one there [gestures].

155. Okay. Is the size the same as a while ago or has it changed? 
156. «... 9 " " Let's say that ... now, I am more inclined to imagine them as pictures, as in "Amicalement vôtre"10, at the beginning, when you see the ... well, the photos of the two ... the two main actors scrolling, when it stops on uh ... two images. They are not compared, they are just shown. And now, I see them like that ... two images, one beside the other.

157. Are they both as clear or is there one that you see better?

158. Well, I see this one far better than the other one.

159. The one you chose?

160. Yes.

Second presentation of the photo

161. Okay. I propose that you return the photo, and say what comes to you.

162. «... It's not the one I chose.

${ }^{10}$ The French translation of the TV series "The Persuaders". 